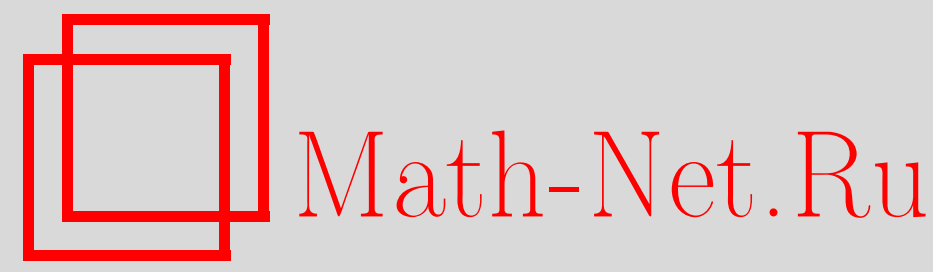

Ю. Г. Прохоров, Степень трехмерных многообразий Фано с каноническими горенштейновыми особенностями, Матем. сб., 2005, том 196, номер 1, 81-122

DOI: https://doi.org/10.4213/sm1262

Использование Общероссийского математического портала Math-Net.Ru подразумевает, что вы прочитали и согласны с пользовательским соглашением

http://www.mathnet.ru/rus/agreement

Параметры загрузки:

IP: 3.80 .253 .173

26 апреля 2023 г., 04:05:40 
УДК 512.7

\author{
Ю.Г. Прохоров
}

\title{
Степень трехмерных многообразий Фано с каноническими горенштейновыми особенностями
}

Рассматриваются трехмерные многообразия $\Phi$ ано $X$ с каноническими горенштейновьми особенностями. Доказана точная оценка степени $-K_{X}^{3} \leqslant 72$.

Библиографияя: 37 названий.

\section{§1. Введение}

Настоящая статья посвящается памяти Андрея Николаевича Тюрина, с которым автор обсуждал рассматриваемые здесь проблемы в 2002 году.

В работе изучаются трехмерные многообразия Фано с каноническими горенштейновьми особенностями. Такие многообразия естественно появляются в теории минимальных моделей, что подтверждает следующий результат Алексеева:

Теорема 1.1 (см. [1]). Пусть $Y$ - трехмерное многообразие $\mathbb{Q}$-Фано с терминальнылми $\mathbb{Q}$-факториальными особенностями и числом Пикара 1 . Если его антиканоническая модель $\Phi_{\left|-K_{Y}\right|}(Y)$ трехмерна, то $Y$ бирачионально изоморфно многообразию Фано $V$ с каноническими горенштейновыми особенностями и свободной от базисных точек линейной системой $\left|-K_{V}\right|$.

Дж. Фано [2], [3] изучал алгебраические многообразия $V \subset \mathbb{P}^{n}$ с общими линейными сечениями $V \cap \mathbb{P}^{n-2}$ - каноническими кривыми. При некоторых дополнительных ограничениях они являются также многообразиями Фано с каноническими горенштейновыми особенностями (см. [4]).

Из основного результата работы [5] следует, что степень многообразий Фано с каноническими горенштейновыми особенностями ограничена. Явная оценка $-K^{3} \leqslant 184$ была получена Чельцовым [6], однако она довольно далека от точной.

ГиПоТЕЗА 1.2 (Фано, Исковских). Пусть $V$ - трехмерное многообразие Фано с каноническими горенитейновыми особенностями. Тогда $-K_{V}^{3} \leqslant 72$.

Точная оценка степени была известна при дополнительном ограничении на особенности.

Работа выполнена при частичной поддержке Российского фонда фундаментальных исследований (грант № 02-01-00441), Программы поддержки ведущих научных школ РФ (гранты № № НШ-489.2003.1, НШ-1910.2003.1) и Программы РАН “Современные проблемы теоретической математики".

(C) Ю.Г. Прохоров 2005 
Теорема 1.3 (см. [7]). Пусть $V$ - трехмерное многообразие Фано с горенштейновыми терминальными особенностями. Тогда $X$ может быть деформировано в неособое многообразие Фано. В частности, $-K_{V}^{3} \leqslant 64$.

Более того, метод векторных расслоений Мукая может быть применен в этом случае и позволяет классифицировать многообразия Фано с терминальными горенштейновыми особенностями [8]. В каноническом случае ситуация вьплядит несколько сложнее: утверждение теоремы 1.3 перестает быть верным, что показывает следующий пример.

Пример 1.4 (ср. [2], [9; гл. 4, замечание 4.2]). Взвешенные проективные пространства $\mathbb{P}(3,1,1,1)$ и $\mathbb{P}(6,4,1,1)$ являются многообразиями Фано с каноническими горенштейновыми особенностями. Для них $-K^{3}=72$, поэтому они не могут быть продеформированы в неособые.

Основной результат настояшей работы - следуюшая теорема.

Тоерема 1.5. Пусть $V$ - трехмерное многообразие Фано с каноническими горенштейновыми особенностями. Тогда $-K_{V}^{3} \leqslant 72$. Более того, из равенства $-K_{V}^{3}=72$ следует, ито многообразие $V$ изоморфно одному из двух взвешенных проективных пространств в примере 1.4.

Таким образом, настояшая работа полностью доказывает гипотезу Фано-Исковских. Из наших результатов немедленно получается следующее

СлЕДСтвиЕ 1.6. Пусть $V \subset \mathbb{P}^{n}$ - нормальное проективное трехмерное многообразие такое, что выполнено следующее свойство:

(†) общее гиперплоское сечение $V \cap \mathbb{P}^{n-1}$ является поверхностью типа $\mathrm{K} 3$ c, возможно, дювалевскими особенностями.

Eсли $\operatorname{deg} V>72$, то $V$ является конусом.

Действительно, согласно [4] (см. также [10]) многообразие $V$ горенштейново и антиканонический класс $-K_{V}$ высекается гиперплоским сечением. Если особенности $V$ рациональны, то они каноничны и по теореме $1.5 \operatorname{deg} V=-K_{V}^{3} \leqslant 72$. Если же множество нерациональных особенностей непусто, то оно нульмерно и из результата [11] получаем, что $V$ - конус.

Аналогично имеем

СлЕДСТвИЕ 1.7. Пусть $U \subset \mathbb{P}^{n}$ - нормальное проективное трехмерное многообразие такое, что выполнено следующее свойство:

(†) общее гиперплоское сечение $U \cap \mathbb{P}^{n-1}$ является поверхностью Энриквеса с, возможно, дювалевскими особенностями.

Если $\operatorname{deg} U>36$, то $U$ является конусом.

Действительно, предположим, что $U$ не является конусом. Тогда согласно [10] (ср. [12]) многообразие $U \mathbb{Q}$-горенштейново, имеет лишш канонические особенности и его антиканонический дивизор Вейля $-K_{U} \mathbb{Q}$-линейно эквивалентен классу гиперплоского сечения $H$. Это означает, что $n\left(K_{U}+H\right) \sim 0$ для некоторого $n \in \mathbb{N}$. 
Дивизор $K_{U}+H$ задает глобальное $n$-листное накрытие $\pi: V \rightarrow U$, неразветвленное над неособой частью $U$. Тогда многообразие $V$ удовлетворяет условиям теоремы 1.5 и поэтому $-K_{V}^{3} \leqslant 72$. Отсюда $\operatorname{deg} U=-K_{U}^{3}=-K_{V}^{3} / n \leqslant 36$.

Отметим, что в отличие от следствия 1.6 оценка в следствии 1.7 не является строгой. Например, несложно показать, что на многообразиях из примера 1.4 инволюция, фактор по которой имеет лишь канонические особенности, не может действовать свободно в коразмерности 2 . Поэтому $\operatorname{deg} U \neq 36$.

Приведем еще одно следствие из нашей теоремы.

СлЕДСТвИЕ 1.8. Пусть $X$ - трехмерное многообразие Фано с каноническими (необязательно горенштейновыми) особенностями. Предположим, ито

(i) образ $\Phi_{\left|-K_{X}\right|}(X)$ отображения, заданного антиканонической линейной системой, трехмерен;

(ii) общий әлемент $F \in\left|-K_{X}\right|$ неприводим и имеет лищъ дювалевские особенности.

Тогда $\operatorname{dim}\left|-K_{X}\right| \leqslant 38$ и эта оценка является строгой.

ДокАЗАТЕЛЬство. Следствие доказывается так же, как и следствие 4.6 в [1]: рассматривается терминальная $\left(K_{X}+\left|-K_{X}\right|\right)$-крепантная модификация

$$
f:\left(Y, \mathscr{L}_{Y}\right) \rightarrow\left(X,\left|-K_{X}\right|\right)
$$

пары $\left(X,\left|-K_{X}\right|\right)$. Тогда линейная система $\mathscr{L}_{Y} \subset\left|-K_{Y}\right|$ свободна и задает морфизм $g: Y \rightarrow \mathbb{P}^{N}$ с факторизацией Штейна $Y \rightarrow V \rightarrow g(Y)$, где $V$ - многообразие Фано с каноническими горенштейновыми особенностями. По нашей теореме $\operatorname{dim}\left|-K_{X}\right| \leqslant \operatorname{dim}\left|\mathscr{L}_{Y}\right| \leqslant \operatorname{dim}\left|-K_{V}\right| \leqslant 38$.

Последнее следствие является аргументом в пользу гипотезы о том, что оценка из теоремы 1.5 имеет место для всех трехмерных многообразий Фано с каноническими особенностями (Х. Такаги).

В заключение отметим, что в случае, когда многообразие $V$ имеет лишш особенности типа $c D V$, оценка $-K_{V}^{3} \leqslant 72$ не является точной (поскольку многообразия из примера 1.4 имеют особенности хуже, чем cDV). Мы ожидаем в этом случае лучшую оценку $-K_{V}^{3} \leqslant 64$. Доказательство может быть получено незначительной модификацией нашего метода. С другой стороны, следуюшая гипотеза выглядит вполне правдоподобной:

ГиПотеЗА 1.9 (ср. теорема 1.3). Пусть $V$ - трехмерное многообразие Фано, имеющее лишь особенности типа $c D V$. Тогда $V$ допускает сглаживание при помощи плоских деформаций. В частности, $-K_{V}^{3} \leqslant 64$.

ЗАмЕчАнИЕ. Вопросы о рациональности некоторых многообразий Фано, удовлетворяюших условию теоремы 1.5, обсуждался в [13].

ИДЕЯ ДОКАЗАТЕЛЬСТВА ТЕОРЕМЫ 1.5. ПроилЛюстрируем идею доказательства теоремы 1.5 на ее двумерном аналоге, а именно передокажем хорошо известный факт: степень поверхности дель Пецшо $V$ с дювалевскими особенностями не 
превосходит 8 (если $V$ действительно особа). Пусть $V$ - поверхность дель Пеццо с дювалевскими особенностями степени $K_{V}^{2}=d$. Предположим, что $V$ особо. Если $d \geqslant 3$, то антиканоническая линейная система задает вложение $V \hookrightarrow \mathbb{P}^{d}$. Рассмотрим линейную систему $\mathscr{H} \subset\left|-K_{V}\right|$ гиперплоских сечений, проходящих через особую точку $P \in V$. Пусть $\phi: W \rightarrow V$ - минимальное разрешение. Тогда $\phi^{*}\left(K_{V}+\mathscr{H}\right)=K_{W}+\mathscr{H}_{W}+B$, где $\mathscr{H}_{W}-$ собственный прообраз $\mathscr{H}$, а $B$ - ненулевой эффективный дивизор. Применим программу минимальных моделей относительно логдивизора $K_{W}+\mathscr{H}_{W}$. Так как линейная система $\mathscr{H}_{W}$ не имеет неподвижных компонент, то мы останемся в категории неособых поверхностей. В конце мы получим пару $\left(X, \mathscr{H}_{X}\right)$, на которой имеется небирациональное $\left(K_{X}+\mathscr{H}_{X}\right)$-отрицательное стягивание. Имеются две возможности:

a) $X \simeq \mathbb{P}^{2}$, тогда $d-1=\operatorname{dim} \mathscr{H}=\operatorname{dim} \mathscr{H}_{X} \leqslant 5$;

б) $X \simeq \mathbb{F}_{n}$, тогда $\mathscr{H}_{X}$ - линейная система сечений проекции $\mathbb{F}_{n} \rightarrow \mathbb{P}^{1}$.

Отметим, что бирациональный прообраз линейной системы $\left|-K_{V}\right|$ содержится в $\left|-K_{X}\right|$. В частности, $\left|-K_{X}\right|$ не имеет неподвижных компонент. В случае б) это возможно только при $n \leqslant 2$ и тогда $d=\operatorname{dim}\left|-K_{V}\right| \leqslant \operatorname{dim}\left|-K_{X}\right| \leqslant 8$.

Наше доказательство в трехмерном случае следует приведенному вьше. Однако оно гораздо сложнее из-за многочисленных технических деталей. Поясним теперь подробнее трехмерньй случай.

ОПРЕДЕЛЕНИЕ 1.10. Проективное алгебраическое многообразие $W$ называется многообразием почти Фано, если его антиканонический дивизор является численно эффективным объемньм дивизором $\mathbb{Q}$-Картье.

Пусть $V$ - трехмерное многообразие Фано с каноническими горенштейновыми особенностями. Рассмотрим его терминальную $\mathbb{Q}$-факториальную модификацию $\phi: W \rightarrow V$ (см. предложение 3.5). Здесь $K_{W}=\phi^{*} K_{V}$ и $W$ имеет лишь терминальные $\mathbb{Q}$-факториальные горенштейновы особенности. Согласно [14; лемма 5.1] любой дивизор Вейля на $W$ является дивизором Картье, т.е. $W$ факториально. Обратно, для каждого трехмерного многообразия почти Фано $W$ с терминальными факториальньми особенностями его кратный антиканонический образ $V=$ $\Phi_{\left|-n K_{W}\right|}(W)$ для некоторого $n \in \mathbb{N}$ является многообразием Фано с каноническими горенштейновыми особенностями. При этом $K_{W}=\Phi^{*} K_{V}$ и $-K_{V}^{3}=-K_{W}^{3}$. Таким образом, оценка в теореме 1.5 эквивалентна аналогичной оценке степени многообразий почти Фано $W$ с терминальными факториальньми особенностями. На таких многообразиях полностью классифицированы $K$-отрицательные экстремальные лучи [15]. Несложный анализ структуры экстремальных лучей показывает, что для $W$, имеющего максимальную степень $\geqslant 72$, соответствующее многообразие $V$ особо вдоль прямой или содержит плоскость. Далее, как и в двумерном аналоге, рассмотренном выше, ограниченность степени $V$ получается детальньм анализом линейной системы гиперплоских сечений, проходящих через эту прямую или плоскость.

Работа была задумана во время участия автора в конференции памяти Дж. Фано (Турин, 2002 г.) и в основном завершена в Исследовательском институте математических наук г. Киото (RIMS) в 2002-2003 гг. Автор благодарен организаторам 
конференции за приглашение и институту RIMS за поддержку и прекрасные условия для работы. Автор также выражает благодарность Х. Такаги, Д. Орлову, И. Чельцову, П. Жанке и И. Радлофу за некоторые полезные обсуждения.

\section{§2. Обозначения, соглашения и предварительные результаты}

В этой статье мы работаем над полем комплексных чисел $\mathbb{C}$. Все многообразия Фано обычно считаются трехмерными, однако леммы 4.2 и 4.3 верны в произвольной размерности по модулю программы минимальных моделей (ПММ). Всегда, когда мы говорим, например, что многообразие имеет канонические особенности, это означает, что особенности не хуже, чем таковые. Обычно мы не делаем различия между дивизорами Картье и соответствующими обратимыми пучками.

ОБОзНА ЧЕния. Через $\mathbb{F}_{n}$ мы будем обозначать рациональную линейчатую поверхность (поверхность Хирцебруха), а через $\Sigma=\Sigma_{n}$ и $l=l_{n}$ - ее минимальное сечение и слой соответственно. При $n \geqslant 1$ существует стягивание $\mathbb{F}_{n} \rightarrow \mathbb{W}_{n}$ минимального сечения $\Sigma$, где $\mathbb{W}_{n}-$ конус над рациональной нормальной кривой степени $n$ в $\mathbb{P}^{n}$. Вершина этого конуса будет обозначаться через $O=O_{n}$.

Всюду далее мы предположим, что $V$ - трехмерное многообразие Фано с каноническими горенштейновьми особенностями. Воспользовавшись теоремой Римана-Роха и теоремой Каваматы-Фивега об обрашении в нуль, несложно найти размерность антиканонической линейной системы (см. [16]):

$$
\operatorname{dim}\left|-K_{V}\right|=-\frac{1}{2} K_{V}^{3}+2
$$

Обозначим $g:=-\frac{1}{2} K_{V}^{3}+1$. Это число называется $р о д о м ~ V$. Таким образом,

$$
-K_{V}^{3}=2 g-2 \text { и } \operatorname{dim}\left|-K_{V}\right|=g+1 .
$$

Tеорема 2.1 (см. [16]). Общий әлемент $L \in\left|-K_{V}\right|$ имеет лишь дювалевские особенности.

Следуюшее предложение несложно выводится из теоремы 2.1 и соответствующих фактов о поверхностях типа К3 [17].

ПРЕДЛОЖЕНИЕ 2.2 (см. [9; гл. 1, предложение 6.1], [18]). В обозначениях, приведенных выше, предположим, что $\mathrm{Bs}\left|-K_{V}\right| \neq \varnothing$. Тогда имеет место один из следующих случаев:

(i) схема $\mathrm{Bs}\left|-K_{V}\right|=C$ является (приведенной) неособой рациональной кривой и общий дивизор $L \in\left|-K_{V}\right|$ также неособ вдоль $C$; в частнос$m u, C \cap \operatorname{Sing} V=\varnothing ;$

(ii) $\mathrm{Bs}\left|-K_{V}\right|=\{P\}$ является точкой, общий дивизор $L \in\left|-K_{V}\right|$ имеет в $P$ обыкновенную двойную особенность и $P \in \operatorname{Sing} V$ (в этом случае $\left.-K_{V}^{3}=2\right)$. 
ПрЕДЛОЖЕНИЕ 2.3 ([6], ср. [9; гл. $1, \S 6]$, см. также [19]). Если $\mathrm{Bs}\left|-K_{V}\right| \neq \varnothing$, $m o-K_{V}^{3} \leqslant 46$.

Аналогично неособому случаю (см. [9]) можно легко доказать следующее

ПРЕДЛОЖЕНИЕ 2.4. В обозначениях, приведенных выше, пусть $\Phi=\Phi_{\left|-K_{V}\right|}$ : $V \rightarrow \bar{V} \subset \mathbb{P}^{g+1}$ - антиканоническое отображение, где $\bar{V}=\Phi(V)$. Тогда $\operatorname{dim} \bar{V} \geqslant 2 u \operatorname{dim} \bar{V}=2$, если и только если $\mathrm{Bs}\left|-K_{V}\right| \neq \varnothing$. Если $\mathrm{Bs}\left|-K_{V}\right|=\varnothing$, то имеет место один из следующих случаев:

(i) $\Phi: V \rightarrow \bar{V}$ является двойньцм накрытием, в этом случае $\bar{V} \subset \mathbb{P}^{g+1}$ многообразие степени $g-1$;

(ii) $\Phi: V \rightarrow \bar{V}$ является изоморфизмом.

ПРЕДЛОЖЕНИЕ 2.5 ([6], ср. [9; гл. $2, \S 2])$. В случае (i) предложсения 2.4 имеет место оченка $-K_{V}^{3} \leqslant 40$.

\section{§3. Некоторые сведения из теории минимальных моделей}

Основные определения и факты из теории минимальных моделей могут быть найдены в многочисленных обзорах и учебниках (см., например, [20]). Здесь мы приведем лишь те факты, которые пока не входят в большинство обзоров.

Особенности линейных систем [1]. Пусть $X$ - нормальное многообразие, и пусть $\mathscr{H}$ - линейная система (дивизоров Вейля) без неподвижных компонент на $X$. Для любого бирационального отображения $\psi: X \rightarrow Y$ через $\mathscr{H}_{Y}$ будет обозначаться собственный (бирациональньй) прообраз $\psi_{*} \mathscr{H}$ линейной системы $\mathscr{H}$. Иногда, если это не приводит к путанице, мы будем писать $\mathscr{H}$ вместо $\mathscr{H}_{Y}$. Предположим, что $K_{X}+H-$ дивизор $\mathbb{Q}$-Картье для $H \in \mathscr{H}$. Для любого хорошего разрешения особенностей $f: Y \rightarrow X$ пары $(X, \mathscr{H})$ мы можем написать

$$
K_{Y}+\mathscr{H}_{Y}=f^{*}\left(K_{X}+\mathscr{H}\right)+\sum_{E} a(E, \mathscr{H}) E
$$

где $E$ пробегает все исключительные дивизоры, а $a(E, \mathscr{H}) \in \mathbb{Q}$. Здесь и ниже в численных формулах мы будем использовать обозначения $\mathscr{H}, \mathscr{H}_{Y}$ и т.д. вместо общих членов этих линейных систем. Говорят, что пара $(X, \mathscr{H})$ имеет канонические особенности (или просто пара $(X, \mathscr{H})$ канонична), если $a(E, \mathscr{H}) \geqslant 0$ для всех $E$. Если общий элемент $\mathscr{H}$ неприводим, то пара $(X, \mathscr{H})$ канонична тогда и только тогда, когда канонична пара $(X, H)$, где $H \in \mathscr{H}$ - общий дивизор [21; теорема 4.8]. В категории трехмерных канонических $\mathbb{Q}$-факториальных пар работает логпрограмма минимальных моделей [1].

Лемма 3.1. Пусть $(X, \mathscr{L})$ - каноническая пара, и пусть $X-\rightarrow Y-\sigma u$ рациональное отображсение, обратное $к$ которому не стягивает дивизоров (бирачиональное 1-стягивание в терминологии ШІокурова). Предположим, что $K_{X}+\mathscr{L} \equiv 0$. Тогда пара $\left(Y, \mathscr{L}_{Y}\right)$ также канонична и $K_{Y}+\mathscr{L}_{Y} \equiv 0$. 
ДокаЗАТЕЛЬСТво. Рассмотрим “домик Хиронаки”

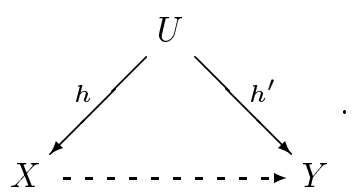

Пусть $\left\{E_{i}\right\}$ - множество всех $h^{\prime}$-исключительных дивизоров. Запишем

$$
\begin{aligned}
& K_{U}+\mathscr{L}_{U}=h^{*}\left(K_{X}+\mathscr{L}_{X}\right)+\sum a_{i} E_{i}, \quad a_{i} \geqslant 0, \\
& K_{U}+\mathscr{L}_{U}=h^{\prime *}\left(K_{Y}+\mathscr{L}_{Y}\right)+\sum b_{i} E_{i} .
\end{aligned}
$$

Тогда $\sum b_{i} E_{i} \overline{\bar{Y}} \sum a_{i} E_{i}$. Это дает нам $a_{i}=b_{i}($ см. [22; 1.1]).

ОПРЕДЕЛЕНИЕ 3.2. Пусть $(X, \mathscr{L})$ и $\left(X^{\prime}, \mathscr{L}^{\prime}\right)$ - логпары. Бирациональный морфизм $f:(X, \mathscr{L}) \rightarrow\left(X^{\prime}, \mathscr{L}^{\prime}\right)$ называется $(K+\mathscr{L})$-крепантныц, если

$$
K_{X}+\mathscr{L}=f^{*}\left(K_{X^{\prime}}+\mathscr{L}^{\prime}\right), \quad \mathscr{L}^{\prime}=f_{*} \mathscr{L}
$$

Бирациональное отображение $(X, \mathscr{L}) \rightarrow\left(X^{\prime}, \mathscr{L}^{\prime}\right)$ называется крепантныл, если существует нормальное многообразие $U$ и коммутативньй треугольник $(K+\mathscr{L})$ крепантных бирациональных морфизмов

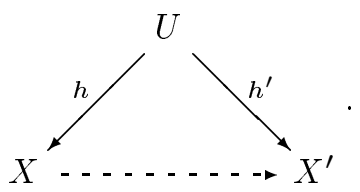

Леммма 3.3 (см. [21; лемма 3.10$])$. Пусть $(X, \mathscr{L})$ - каноническая пара, и пусть $X \rightarrow Y$ - крепантное бирациональное отображение. Тогда пара $\left(Y, \mathscr{L}_{Y}\right)$ также канонична.

Лемма 3.4. Пусть $(W, \mathscr{H})$ - каноническая пара. Предположим, что

(i) многообразие $X$ имеет лишь терминальные $\mathbb{Q}$-факториальные особенности;

(ii) линейная система $\mathscr{H}$ численно әффективна;

(iii) $\mathscr{H}$ составлена из дивизоров Картье.

Тогда шаги $(K+\mathscr{H})$-программы минимальных моделей сохраняют свойства (i)-(iii).

ДоказАТЕЛЬСтво. Пусть $\varphi: W \rightarrow W^{\prime}$ - экстремальное $\left(K_{W}+\mathscr{H}\right)$-отрицательное стягивание, и пусть (как обычно) $\mathscr{H}^{\prime}=\varphi_{*} \mathscr{H}$. Так как линейная система $\mathscr{H}$ численно эффективна, то это стягивание является также и $K_{W}$-отрицательным. Поэтому свойство (i) сохраняется при дивизориальных стягиваниях и флипах. 
Предположим, что стягивание $\varphi$ дивизориально. Тогда многообразие $W^{\prime}$ терминально. Пусть $C$ - любая стягиваемая кривая. Если $\mathscr{H} \cdot C=0$, то по свойствам экстремальных стягиваний $\mathscr{H}=\varphi^{*} \mathscr{H}^{\prime}$ и $\mathscr{H}^{\prime}-$ линейная система численно эффективных дивизоров Картье на $W^{\prime}$. Предположим, что $\mathscr{H} \cdot C \geqslant 1$. Тогда $-K_{W} \cdot C>1$. В частности, $\varphi$ стягивает дивизор $E$ в точку $P^{\prime} \in W^{\prime}$ (см. [23; 2.3]). Поэтому линейная система $\mathscr{H}^{\prime}$ численно эффективна. Действительно, если $R^{\prime} \subset W^{\prime}$ - произвольная кривая и $R \subset W$ - ее собственный прообраз, то $\mathscr{H}^{\prime} \cdot R^{\prime} \geqslant \mathscr{H} \cdot R \geqslant 0$. Так как пары $(W, \mathscr{H})$ и $\left(W^{\prime}, \mathscr{H}^{\prime}\right)$ каноничны, то для общего дивизора $H \in \mathscr{H}$ ограничение $\left.\varphi\right|_{H}: H \rightarrow H^{\prime}=\varphi(H)$ является бирациональным стягиванием нормальных поверхностей с дювалевскими особенностями. По формуле присоединения дивизор $-K_{H}=-\left(K_{W}+H\right)$ является $\left.\varphi\right|_{H^{-о б и л ь н ы м . ~}}$ Поэтому дискрепантности всех исключительных над $P^{\prime} \in H^{\prime}$ дивизоров строго положительны [20; лемма 3.38]. Это означает, что $P^{\prime} \in H^{\prime}$ - неособая точка. Следовательно, неособа также точка $P^{\prime} \in W^{\prime}$ (см. [22; следствие 3.7]) и $H^{\prime}$ является дивизором Картье в $P^{\prime}$.

Предположим, что стягивание $\varphi$ - малое (флипповое). Рассмотрим фолип

$$
W \stackrel{\varphi}{\longrightarrow} W^{\prime} \stackrel{\psi}{\longleftarrow} W^{+}
$$

Пусть $C$ - исключительная кривая. Согласно $[23 ; 2.3]$ имеем $-K_{W} \cdot C<1$. Поэтому $\mathscr{H} \cdot C=0$. Как и выше, по свойствам экстремальных стягиваний $\mathscr{H}=\varphi^{*} \mathscr{H}^{\prime}$ и $\mathscr{H}^{\prime}$ - линейная система численно эффективных дивизоров Картье на $W^{\prime}$. Следовательно, свойства (ii), (iii) вьполняются и для $\mathscr{H}^{+}=\psi^{*} \mathscr{H}^{\prime}$.

\section{Терминальная модификация.}

ПРЕДЛОЖЕНИЕ-ОПРЕДЕЛЕНИЕ 3.5 (см. [24], [23], [20; теоремы 6.23, 6.25]). Пусть $X$-многообразие с каноническими особенностями. Тогда существует многообразие $Y$ с терминальными $\mathbb{Q}$-факториальными особенностями и бирачиональное стягивание $f: Y \rightarrow X$ такое, что $K_{Y}=f^{*} K_{X}$. Такое $f$ назъвается терминальной $\mathbb{Q}$-факториальной модификацией $X$.

\section{$\S 4$. Стягивания экстремальных лучей на трехмерных многообразиях почти Фано}

Для работы с многообразиями Фано очень удобно следующее определение, которое было введено в неопубликованном препринте В. В. Шокурова и автора.

ОПРЕДЕЛЕНИЕ 4.1. 0-парой мы называем пару $(X, D)$, состоящую из проективного алгебраического многообразия $X$ и гранищы $D$ на $X$ таких, что

(i) $(X, D)$ имеет логтерминальные по Кавамате особенности;

(ii) $K_{X}+D \equiv 0$.

Будем говорить, что 0-пара $(X, D)$ порождающая, если $X \mathbb{Q}$-факториально и компоненты границы $D$ порождают группу $N^{1}(X) \mathbb{R}$-дивизоров по модулю численной эквивалентности. Следующие две леммы являются легкими следствиями логпрограммы минимальных моделей. 
Лемма 4.2 (см. [25]). Пусть (X,D) - порождающая 0-пара.

(i) Cуществует граница $\Delta$ такая, что пара $(X, \Delta)$ логтерминальна по Кавамате, дивизор $-\left(K_{X}+\Delta\right)$ обилен $и \operatorname{Supp}(\Delta)=\operatorname{Supp}(D)$.

(ii) Конус Мори $\overline{\mathrm{NE}}(X)$ полиэдрален и пороэсдается стягиваемыми әкстремальными лучами (т.е. лучами $R$, для которых существует стягивание $\varphi_{R}: X \rightarrow X^{\prime}$ в смысле Мори [26], морфизм $\varphi_{R}$ нормальных многообразий со связными слоями такой, что образ кривой $C$ является точкой тогда и только тогда, когда $[C] \in R)$.

(iii) Ө-программа минимальных моделей работает относительно любого (необязательно әффективного) дивизора $\Theta$.

ЛЕмма 4.3 (см. [25]). Пусть $(X, \Delta)$ - $\mathbb{Q}$-факториальная логтерминальная по Кавамате пара такая, что дивизор $-\left(K_{X}+\Delta\right)$ численно әффективен и объемен. Тогда существует гранища $D$, относительно которой $(X, D)$ порожсдающая 0-пара.

Лемма 4.4. Пусть $(X, D)$ - порождающая 0-пара, и пусть $M-$ цельй численно эффективный дивизор Вейля на $X$. Тогда $H^{i}(X, M)=0$ для $i>0$.

ДоказАТЕльство. По лемме 4.2 существует граница $\Delta$ такая, что пара $(X, \Delta)$ логтерминальна по Кавамате и дивизор $-\left(K_{X}+\Delta\right)$ обилен. Далее утверждение следует из теоремы Каваматы-Фивега об обрашении в нуль.

Пусть $R \subset \overline{\mathrm{NE}}(X)$ - (необязательно $K$-отрицательный) экстремальньй луч на нормальном проективном многообразии $X$. Положим

$$
\operatorname{Ex}(R)=\bigcup_{[C] \in R} C
$$

Будем говорить, что стягиваемый экстремальный луч $R$ имеет тип $(n, m)$, если $\operatorname{dim} \operatorname{Ex}(R)=n$ и $\operatorname{dim} \varphi_{R}(\operatorname{Ex}(R))=m$. Далее мы различим случаи $(n, m)^{-},(n, m)^{+}$ и $(n, m)^{0}$ в зависимости от знака $K_{X} \cdot R$.

Всюду далее мы будем предполагать, что $W$ - многообразие почти Фано с $\rho(W)>1$, имеющее лиш терминальные факториальные особенности. Согласно лемме 4.3 все экстремальные лучи на $W$ стягиваемы. Антиканонический дивизор $-K_{W}$ определяет грань конуса Мори $\overline{\mathrm{NE}}(W)$ и ее стягивание $\phi: W \rightarrow V$ на многообразие Фано с каноническими горенштейновыми особенностями.

Рассмотрим бирациональное $K$-отрицательное стягивание $f: W \rightarrow W^{\prime}$. Согласно $[23 ;(2.3 .2)]$ оно дивизориально.

ПРЕДЛОЖЕНИЕ-ОПРЕДЕЛЕНИЕ 4.5. В обозначениях, приведенных выше, пусть $S$ - исключительньи ди диизор. Если $f: W \rightarrow W^{\prime}$ mипа $(2,0)^{-}$, то имеет место один из следуюших случаев:

Случай $(2,0)_{\bullet}^{-}$. $W^{\prime}$ такэсе имеет терминальные факториальные особенности и является многообразием почти Фано с $-K_{W^{\prime}}^{3} \geqslant-K_{W}^{3}$.

Случай $(2,0)_{\circ}^{-} . \quad S \simeq \mathbb{P}^{2}, \mathscr{O}_{S}(S) \simeq \mathscr{O}_{\mathbb{P}^{2}}(2)$ и $f(S) \in W^{\prime}-$ mочка mипа $\frac{1}{2}(1,1,1)$. 
Если $f: W \rightarrow W^{\prime}$ типа $(2,1)^{-}$, то $W^{\prime}$ также имеет терминальные факториальнье особенности и имеет место один из следующих случаев.

Случай $(2,1)_{\bullet}^{-}$. $W^{\prime}-$ также многообразие почти Фано $c-K_{W^{\prime}}^{3} \geqslant-K_{W}^{3}$.

Случай $(2,1)_{\circ}^{-}$. Для $C:=f(S)$ имеем $K_{W^{\prime}} \cdot C>0$ и $C$-единственная кривая, положительно пересекающая $K_{W^{\prime}}$. B этом случае существует малое стягивание кривой $C, C \simeq \mathbb{P}^{1} u W^{\prime}$ неособо вдоль $C$. Можно выделить два подслучая:

$$
\begin{array}{ll}
(2,1)_{\circ 0}^{-} . & S \simeq \mathbb{P}^{1} \times \mathbb{P}^{1} ; \\
(2,1)_{\circ 1}^{-} . & S \simeq \mathbb{F}_{1} .
\end{array}
$$

ДокАЗАТЕЛЬСтво. В случае $(2,0)^{-}$утверждение следует из классификации экстремальных лучей на трехмерных многообразиях с терминальньми факториальными особенностями [15].

Рассмотрим случай $(2,1)^{-}$. Согласно [15] многообразие $W^{\prime}$ неособо вдоль $C$ и $f$ - раздутие $C$. Имеем $K_{W}=f^{*} K_{W^{\prime}}+S$ и

$$
K_{W}^{3}=K_{W^{\prime}}^{3}+3 f^{*} K_{W^{\prime}} \cdot S^{2}+S^{3} .
$$

Если $W^{\prime}$ - многообразие почти Фано, то

$0 \leqslant\left(-K_{W}\right)^{2} \cdot S=2 f^{*} K_{W^{\prime}} \cdot S^{2}+S^{3}, \quad 0 \leqslant\left(-K_{W}\right) \cdot\left(-f^{*} K_{W^{\prime}}\right) \cdot S=f^{*} K_{W^{\prime}} \cdot S^{2}$.

Это дает нам, что $K_{W}^{3} \geqslant K_{W^{\prime}}^{3}$.

Предположим теперь, что $W^{\prime}$ не является многообразием почти Фано. Тогда $K_{W^{\prime}} \cdot \Gamma>0$ для некоторой неприводимой кривой $\Gamma$. Пусть $\widetilde{\Gamma} \subset W-$ неприводимая кривая, доминируюшая $\Gamma$. Так как $f^{*} K_{W^{\prime}} \cdot \widetilde{\Gamma}>0$ и $K_{W} \cdot \widetilde{\Gamma} \leqslant 0$, то $S \cdot \widetilde{\Gamma}<0$. Следовательно, $\Gamma=C$. По лемме 4.3 существует гранища $D$, для которой $(W, D)-$ порождаюшая 0-пара. Пусть $D^{\prime}:=f_{*} D$. Так как $K_{W}+D \sim 0$, то $K_{W^{\prime}}+D^{\prime} \sim 0$ и $\left(W^{\prime}, D^{\prime}\right)$ - также 0-пара. Далее, $D^{\prime} \cdot C<0$ и $C$ - единственная неприводимая кривая с этим свойством. По лемме 4.2 кривая $C$ порождает экстремальный луч и соответствуюшее стягивание малое. Из теоремы Каваматы-Фивега об обрашении в нуль немедленно выводится, что $C \simeq \mathbb{P}^{1}$ (см. [23; следствие 1.3$]$ ).

Отсюда $S \simeq \mathbb{F}_{n}$ для некоторого $n \geqslant 0$. Далее,

$$
K_{W}^{2} \cdot S=-K_{W^{\prime}} \cdot C+2-2 p_{a}(C)=2-K_{W^{\prime}} \cdot C .
$$

Ясно, что ограничение $-\left.K_{W}\right|_{S}$ является сечением расслоения $S \rightarrow C$. Пусть $\Sigma-$ минимальное сечение, и пусть $l$ - слой. Тогда мы можем записать

$$
-\left.K_{W}\right|_{S} \sim \Sigma+(n+a) l .
$$

Так как этот дивизор численно эффективен, то $a \geqslant 0$. Таким образом,

$$
0 \leqslant-K_{W^{\prime}} \cdot C=K_{W}^{2} \cdot S-2=\left(-\left.K_{W}\right|_{S}\right)^{2}-2=n+2 a-2 .
$$

Это дает нам, что $n+2 a<2$, т.е. $a=0$ и $n \leqslant 1$. 
СлЕДСтвиЕ 4.6. В обозначениях, приведенных выше, имеем следующую табличу:

\begin{tabular}{|c|c|c|c|c|c|}
\hline & $S$ & $N_{C / W^{\prime}}$ & $K_{W^{\prime}} \cdot C$ & $K_{W} \cdot \Sigma$ & $-K_{W^{\prime}}^{3}$ \\
\hline$(2,1)_{\circ 0}^{-}$ & $\mathbb{P}^{1} \times \mathbb{P}^{1}$ & $\mathscr{O}(-2) \oplus \mathscr{O}(-2)$ & 2 & 0 & $-K_{W}^{3}-2$ \\
$(2,1)_{\circ 1}^{-}$ & $\mathbb{F}_{1}$ & $\mathscr{O}(-1) \oplus \mathscr{O}(-2)$ & 1 & 0 & $-K_{W}^{3}$ \\
\hline
\end{tabular}

ДокАЗАТЕЛЬСтво. В наших двух случаях $-\left.K_{W}\right|_{S} \sim \Sigma+n l$, см. (4.1). Из (4.2) получаем, что $-K_{W^{\prime}} \cdot C=n-2$. Отсюда

$$
\operatorname{deg} N_{C / W^{\prime}}=-K_{W^{\prime}} \cdot C-2=n-4 .
$$

Таким образом,

$$
\begin{aligned}
K_{W}^{3} & =K_{W^{\prime}}^{3}+3 f^{*} K_{W^{\prime}} \cdot S^{2}+S^{3}=K_{W^{\prime}}^{3}-3 K_{W^{\prime}} \cdot C+2+K_{W^{\prime}} \cdot C \\
& =K_{W^{\prime}}^{3}-2 K_{W^{\prime}} \cdot C+2=K_{W^{\prime}}^{3}+2(n-2)+2=K_{W^{\prime}}^{3}+2 n-2 .
\end{aligned}
$$

СЛЕДСТВИЕ 4.7. В случае $(2,1)_{\text {о0 }}^{-}$образ $\phi(S)$ исключительного дивизора на $V$ - прямая (рациональная кривая $\Gamma$ такая, что $-K_{V} \cdot \Gamma=1$ ) и многообразие $V$ особо вдоль $\phi(S)$.

В случаях $(2,1)_{\circ 1}^{-}$и $(2,0)_{\circ}^{-}$образ $\phi(S)$ - плоскость (рачиональная поверхность П такая, что $\left.K_{V}^{2} \cdot \Pi=1\right)$.

Теперь мы рассмотрим стягивания типа $(2,1)^{0}$.

Лемма 4.8. Пусть $X$ - трехмерное многообразие $с$ терминальными факториальными особенностями, и пусть $f: X \rightarrow X^{\prime}$ - экстремальное стягивание типа $(2,1)^{0}$. Тогда многообразие $X^{\prime}$ 2-факториально (последнее означает, что для любого челого дивизора $G$ на $X^{\prime} 2 G$ - дивизор Картье).

ДокаЗАтЕЛЬСтво. Рассмотрим общее гиперплоское сечение $H^{\prime} \subset X^{\prime}$, и пусть $H \subset X$ - собственный прообраз $H^{\prime}$. Зафиксируем точку $P \in X^{\prime} \cap f(E)$. Мы можем считать, что $X^{\prime}$ - достаточно малая окрестность $P$. Тогда $P \in H^{\prime}-$ дювалевская особенность, а $\left.f\right|_{H}: H \rightarrow H^{\prime}$ - ее минимальное разрешение. Рассмотрим также общее сечение $F^{\prime} \subset X^{\prime}$, проходящее через $f(E)$. Запишем $f^{*} F^{\prime}=F+r E$, где $F$ собственный прообраз и $r \in \mathbb{N}$. Ограничивая на $H$, получим $f_{H}^{*} F_{H^{\prime}}^{\prime}=F_{H}+r E_{H}$. Здесь $F_{H^{\prime}}^{\prime}$ является общим гиперплоским сечением особенности $P \in H^{\prime}$. Поэтому $\Gamma:=r E_{H}-$ фундаментальный цикл (дювалевской) особенности $P \in H^{\prime}$. Запишем $\Gamma=\sum \gamma_{i} \Gamma_{i}$. Все компоненты $\Gamma_{j}$ численно пропорциональны на $X$. В частности, все $\Gamma_{j} \cdot \Gamma$ отличны от нуля, т.е. фундаментальный цикл относительно антиобилен. Предположим, что $P \in H^{\prime}$ - не особенность типа $A_{1}$. Тогда по определению фундаментального цикла $\left(\Gamma-\Gamma_{j}\right) \cdot \Gamma_{j}>0$ для всех $j$. Поэтому $0>\Gamma \cdot \Gamma_{j}>-2$. Отсюда $\Gamma \cdot \Gamma_{j}=-1$ для всех $j$. С другой стороны, $\sum \gamma_{j}=-\Gamma^{2}$ равно кратности рациональной особенности $P \in H^{\prime}$, т.е. $\sum \gamma_{j}=2$. Это возможно, только если $\Gamma=\Gamma_{1}+\Gamma_{2}$ и $P \in H^{\prime}$ - особенность типа $A_{2}$. Таким образом, мы получаем два случая:

(i) $\Gamma=\Gamma_{1}$ и $P \in H^{\prime}-$ особенность типа $A_{1}$;

(ii) $\Gamma=\Gamma_{1}+\Gamma_{2}, \Gamma_{1} \equiv \Gamma_{2}$ и $P \in H^{\prime}$ - особенность типа $A_{2}$. 
Пусть $F^{\prime}$ - целый дивизор на $X^{\prime}$, пусть $F$ - его собственный прообраз на $X$, и пусть $F \cdot \Gamma_{i}=a$. Рассмотрим, например, случай (ii). Тогда $(F+\operatorname{ar} E) \cdot \Gamma_{i}=$ $a+a \Gamma \cdot \Gamma_{i}=0$. По теореме о конусе $F+a r E=f^{*} F^{\prime}$ и $F^{\prime}-$ дивизор Картье. Аналогично, в случае (i) получаем, что $2 F^{\prime}$ - дивизор Картье.

\section{Стягивания на кривую.}

ПРЕДЛОЖЕНИЕ 4.9. Предположим, что на $W$ существует әкстремальный луч типа $(3,2)$. Тогда $-K_{W}^{3} \leqslant 54$.

Лемма 4.10. Предположим, что $\rho(W)=2$ и морфизм $\phi: W \rightarrow V$ стягивает дивизор на кривую. Пусть $S-$ простой дивизор на $W$. Тогда

$$
-K_{W}^{3} \leqslant \min \left(54,4 K_{W}^{2} \cdot S\right)
$$

ДокаЗАТЕЛЬСтво. Положим $\bar{S}:=\phi_{*} S, d:=-K_{W}^{3}, \delta:=K_{W}^{2} \cdot S$ и предположим, что $d>4 \delta$. Так как многообразие $V \mathbb{Q}$-факториально и $\rho(V)=1$, то $d \bar{S} \equiv-\delta K_{V}$. С другой стороны, по лемме 4.8 дивизор $G:=2 \phi_{*} \bar{S}$ является дивизором Картье. Таким образом, $-K_{V} \equiv \frac{d}{2 \delta} G$, где $\frac{d}{2 \delta}>2$. Пусть $H$ - обильная образуюшая групшы $\operatorname{Pic}(V) \simeq \mathbb{Z}$. Тогда $-K_{V}=r H$, где $r \geqslant 3$. Общий элемент $H \in|H|$ является поверхностью дель Пещцо с дювалевскими особенностями (см. [18]). По формуле присоединения $-\left.K_{H} \equiv(r-1) H\right|_{H}$. Отсюда $H \simeq \mathbb{P}^{2}$ или $H-$ квадрика в $\mathbb{P}^{3}$. Отсюда немедленно получается, что $V$ изоморфно $\mathbb{P}^{3}$ или квадрике в $\mathbb{P}^{4}$. Первый случай невозможен, поскольку $V$ особо. Во втором случае мы имеем $-K_{V}^{3}=54$.

ДОКАЗАТЕЛЬСТВО ПРЕДЛОЖЕНИЯ 4.9. ОбщиЙ сЛой $W_{\eta}$ соответствуюшего стягивания является неособой поверхностью дель Пецо. Следовательно, $K_{W}^{2} \cdot W_{\eta}=K_{W_{\eta}}^{2} \leqslant 9$. По лемме 4.10 имеем $-K_{W}^{3} \leqslant 54$.

\section{§5. Случай расслоения на коники}

ПРЕДПОЛОЖЕНИЯ 5.1. На протяжении всего параграфа мы будем считать, что $W$ - многообразие почти Фано с терминальными факториальными особенностями такое, что на $W$ сушествует экстремальное стягивание $f: W \rightarrow Z$ на поверхность (т.е. типа $(3,2))$. Основной результат этого параграфа - следуюшее

ПРЕДЛОЖЕНИЕ 5.2. (i) Поверхность $Z$ неособа и является поверхностью почти дель Пецио (ср. [27], [28]).

(ii) Eсли $f: W \rightarrow Z$ не является $\mathbb{P}^{1}$-расслоением, то $-K_{W}^{3} \leqslant 54$ (ср. [29]).

(iii) Если $f: W \rightarrow Z$ является $\mathbb{P}^{1}$-расслоением, то $-K_{W}^{3} \leqslant 64$ за одним исключением:

$(*)-K_{W}^{3}=72, Z \simeq \mathbb{P}^{2} u W \simeq \mathbb{P}\left(\mathscr{O}_{\mathbb{P}^{2}} \oplus \mathscr{O}_{\mathbb{P}^{2}}(3)\right)$ (см. пример 1.4). 
ДоКАЗАТЕЛЬСтво. (i) Согласно [15] поверхность $Z$ неособа и $W / Z$ - (возможно, особое) расслоение на коники. Пусть $\mathscr{E}:=f_{*} \mathscr{O}_{W}\left(-K_{W}\right)$. Тогда $\mathscr{E}-$ векторное расслоение ранга 3 . Рассмотрим его проективизацию $\mathbb{P}(\mathscr{E})$, и пусть $M-$ тавтологический дивизор на $\mathbb{P}(\mathscr{E})$. Сушествует вложение $W \hookrightarrow \mathbb{P}(\mathscr{E})$ такое, что $-K_{W}=\left.M\right|_{W}$ и каждый слой $W_{z}, z \in Z$, является коникой в слое $\mathbb{P}(\mathscr{E})_{z}$ расслоения (см. [26], [15]).

Положим $L:=f_{*} K_{W}^{2}$. Для $m \gg 0$ дивизор $m^{2} L=f_{*}\left(m K_{W}\right)^{2}$ на $Z$ подвижен и объемен. Имеем стандартную формулу $-4 K_{Z} \equiv f_{*} K_{W}^{2}+\Delta$ (см., например, [27]). Здесь $\Delta-$ дискриминант $f$ (приведенньй дивизор на $Z$ ). Таким образом,

$$
-4 K_{Z} \equiv L+\Delta \text {. }
$$

Предположим, что существует неприводимая кривая $C \subset Z$ такая, что $K_{Z}$. $C>0$. Из (5.1) вытекает, что $\left(4 K_{Z}+\Delta\right) \cdot C=-L \cdot C \leqslant 0$. Следовательно, $\Delta \cdot C<0$ и $C^{2}<0$. Рассмотрим два случая.

$C$ - компонента $\Delta$. Тогда

$-2 \leqslant 2 p_{a}(C)-2=\left(K_{Z}+C\right) \cdot C \leqslant\left(K_{Z}+\Delta\right) \cdot C=-\left(3 K_{Z}+L\right) \cdot C \leqslant 0$.

Так как $K_{Z} \cdot C$ - положительное целое, это дает нам противоречие.

$C$ не является компонентой $\Delta$. Тогда

$-2 \leqslant 2 p_{a}(C)-2=\left(K_{Z}+C\right) \cdot C \leqslant\left(K_{Z}+\Delta+C\right) \cdot C=-\left(3 K_{Z}+L\right) \cdot C+C^{2}<0$.

Как и выше, получаем противоречие. Следовательно, дивизор $-K_{Z}$ численно эффективен. Из (5.1) получаем, что $-K_{Z}$ объемен. Это доказывает (i).

Докажем (ii). Наше доказательство полностью аналогично приведенному в работе [29], где такая же оценка доказывалась для неособых многообразий Фано, представимых в виде расслоений на коники.

Следуюшая лемма хорошо известна, однако автор не смог найти подходяшей ссылки.

Лемма 5.3. Пусть $f: X \rightarrow Z$ - (возможно, особое) расслоение на коники над неособой поверхностью. Предположим, что дискриминантная кривая $\Delta$ - дерево рациональных кривых. Тогда $\Delta=\varnothing$ и $f$ является $\mathbb{P}^{1}$-расслоением (в частности, $X$ неособо).

ДокАЗАТЕЛЬСтво. Согласно [30; теорема 1.13] сушествует стандартная модель, т.е. коммутативная диаграмма

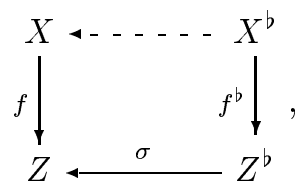

в которой $f^{b}$ - стандартное расслоение на коники, $\sigma$ - композиция раздутий над точками конечного множества $M:=f(\operatorname{Sing}(X))$ и $X^{b} \rightarrow X-$ бирациональное отображение, являющееся изоморфизмомнад $X \backslash f^{-1}(M)$. Дискриминантная кривая $\Delta^{b}$ стандартного расслоения $f^{b}$ содержится в $\sigma^{-1}(\Delta) \cup \sigma^{-1}(M)$ и поэтому также является деревом рациональных кривых. Из точной последовательности Артина-Мамфорда вытекает тогда, что $\Delta^{b}=\varnothing$, т.е. $f^{b}-\mathbb{P}^{1}$-расслоение. 
Лемма 5.4 (ср. [31; $\S 2]$, [32; лемма 4]). Пусть $f: X \rightarrow Z-\mathbb{P}^{1}$-расслоение над неособой проективной поверхностью $Z$. Предположим, что существует $(-1)$-кривая $C \subset Z$, и пусть $\delta: Z \rightarrow Z^{\prime}-$ стяяивание $C$.

(i) Относительный конус Мори $\overline{\mathrm{NE}}\left(X / Z^{\prime}\right)$ порождается классами двух кривых: слоя проекции $f$ и $\Sigma$ - минимального сечения линейчатой поверхности $f^{-1}(C)$.

(ii) Пусть $f^{-1}(C) \simeq \mathbb{F}_{n}$. Тогда $K_{X} \cdot \Sigma=n-1$.

(iii) Eсли $n=0$, то существует коммутативная диаграмма

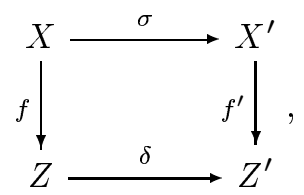

где $\sigma$ - стягивание $f^{-1}(C) \simeq \mathbb{P}^{1} \times \mathbb{P}^{1}$ на вторую образующую, а $f^{\prime}-\mathbb{P}^{1}$-расслоение.

(iv) Eсли $n=1$, то существует коммутативная диаграмма

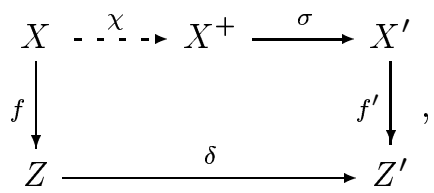

где $\chi$ - флоп, $\sigma$ - экстремальное дивизориальное стягивание дивизора в неособую точку, а $f^{\prime}-\mathbb{P}^{1}$-расслоение.

(v) Если $n \geqslant 1$, то существует коммутативная диаграмма

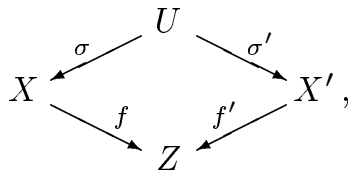

где $\sigma$-раздутие $\Sigma, \sigma^{\prime}$ - стягивание собственного прообраза поверхности $f^{-1}(C)$, a $f^{\prime}-\mathbb{P}^{1}$-расслоение. $B$ этом случае $\sigma^{-1}(C) \simeq \mathbb{F}_{m}$ с $m<n$.

ДокАЗАТЕЛЬСТво. Положим $D:=f^{-1}(C)$. Пункт (i) очевиден, поскольку $\rho\left(X / Z^{\prime}\right)=2$. Пункт (ii) следует из равенств

$-2=\left(K_{D}+\Sigma\right) \cdot \Sigma=\left(K_{X}+D\right) \cdot \Sigma-n=K_{X} \cdot \Sigma+f^{*} C \cdot \Sigma-n=K_{X} \cdot \Sigma+C^{2}-n$.

Для доказательства п. (iii) нужно просто проверить критерий стягиваемости поверхности $D \simeq \mathbb{P}^{1} \times \mathbb{P}^{1}$ на вторую систему образуюших. Докажем п. (iv) (cp. [33; $\S 4.1])$. Так как $K_{X} \cdot \Sigma=0$, то существует флоп $\chi: X \rightarrow X^{+}$с центром в кривой $\Sigma$. Здесь антиканонический дивизор $-K_{X}$ численно эффективен над $Z^{\prime}$, а поэтому то 
же самое верно и для $-K_{X^{+}}$. Конус Мори $\overline{\mathrm{NE}}\left(X^{+} / Z^{\prime}\right)\left(\right.$ как и $\left.\overline{\mathrm{NE}}\left(X / Z^{\prime}\right)\right)$ порождается двумя экстремальными лучами. Один из них - малый и порождается флоп-

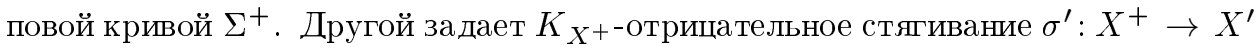
над $Z^{\prime}$. Многообразие $X^{+}$неособо [20; теорема 6.15], а слой проекции $X^{+} \rightarrow Z^{\prime}$ двумерен и содержит $D^{+}-$собственный прообраз поверхности $D$. Легко видеть, что $K_{X^{+}}^{2} \cdot D^{+}=K_{X}^{2} \cdot D($ см. $[33 ; \S 4.1])$. Отсюда

$$
K_{X^{+}}^{2} \cdot D^{+}=\left(K_{X}+D\right)^{2} \cdot D-2\left(K_{X}+D\right) \cdot D^{2}=K_{D}^{2}-\left.2 K_{D} \cdot D\right|_{D}=4 .
$$

Согласно классификации экстремальных лучей на неособых многообразиях [26] морфизм $\sigma^{\prime}$ не может стягивать дивизор в особую точку. Следовательно, $X^{\prime}$ неособо, а так как морфизм $f^{\prime}$ является гладким вне слоя $f^{\prime-1}(\delta(C))$, то он является гладким всюду [26; теорема 3.5$]$. Поэтому $f^{\prime}-\mathbb{P}^{1}$-расслоение. Если $f^{\prime}\left(D^{+}\right)$-кривая, то $\sigma^{\prime}$ - раздутие слоя морфизма $f^{\prime}$. Но тогда $f^{\prime-1}(\delta(C))=D^{+} \simeq \mathbb{P}^{1} \times \mathbb{P}^{1}$ и на $X^{+}$не существует малых стягиваний. Противоречие доказывает п. (iv).

Докажем, наконец, п. (v). Пусть $E$ - исключительный дивизор раздутия $\sigma$, пусть $D_{U}-$ собственный прообраз $D$ на $U$, и пусть $D^{\prime}:=\sigma^{\prime}(E)$. Ясно, что дивизор $D_{U}$ удовлетворяет критерию стягиваемости на кривую. Таким образом, сушествует стягивание $\sigma^{\prime}: U \rightarrow X^{\prime}$ над $Z$, где многообразие $X^{\prime}$ неособо. Так как ограничение $\left.\sigma^{\prime}\right|_{E}: E \rightarrow D^{\prime}$ является изоморфизмом, достаточно доказать, что $E \simeq \mathbb{F}_{m}$ с $m<n$. Для нормального пучка имеем разложение $N_{\Sigma / X}=\mathscr{O}_{\mathbb{P}^{1}}(a) \oplus$ $\mathscr{O}_{\mathbb{P}^{1}}(b), a \geqslant b$. Тогда $m=a-b$. С другой стороны, мы имеем точную последовательность

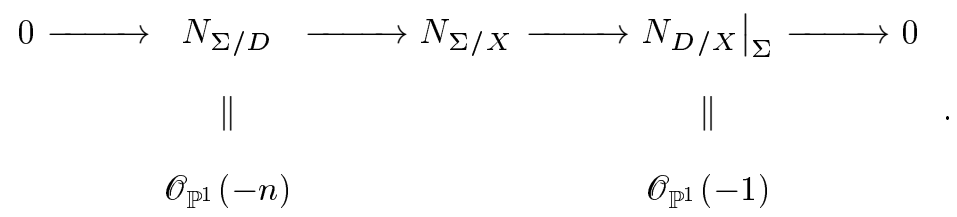

Это дает нам, что

$$
a+b=\operatorname{deg} N_{\Sigma / D}+\left.\operatorname{deg} N_{D / X}\right|_{\Sigma}=-n-1 .
$$

Отсюда $n=-a-b-1$. Если $-n>b$, то в (5.4) проекция $\mathscr{O}_{\mathbb{P}^{1}}(-n)$ на второе слагаемое в $N_{\Sigma / X}$ тривиальна и $-n=a$. Следовательно, $b=-1$ и $m=a-b=$ $1-n<n$. Если же $-n \leqslant b$, то $a+1 \leqslant 0$ и

$$
m=a-b \leqslant-1-b \leqslant-a-b-2=n-1 .
$$

Закончим доказательство леммы 5.3. Согласно лемме 5.4 мы можем постягивать все $(-1)$-кривые в $\sigma^{-1}(M)$. Более точно, существует коммутативная диаграмма

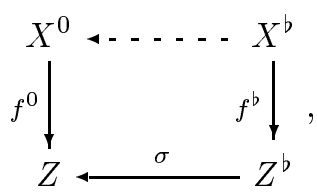


где $f^{0}-\mathbb{P}^{1}$-расслоение, а бирациональное отображение $X^{b} \rightarrow X^{0}$ является изоморфизмом над $X \backslash f^{0-1}(M)$. Следовательно, расслоения на коники $X / Z$ и $X^{0} / Z$ изоморфны в коразмерности 1 . Но тогда они изоморфны всюду, $X$ неособо и $f-$ $\mathbb{P}^{1}$-расслоение.

Таким образом, мы можем считать, что в случае (ii) дискриминантная кривая непуста.

ЛЕмма 5.5. Имеют место следующие соотношения:

(i) $\Delta \sim-3 K_{Z}-c_{1}(\mathscr{E})$;

(ii) $W \sim 2 M+f^{*}\left(-K_{Z}-c_{1}(\mathscr{E})\right)$;

(iii) $-K_{W}^{3}=c_{1}(\mathscr{E}) \cdot\left(-K_{Z}+c_{1}(\mathscr{E})\right)-2 c_{2}(\mathscr{E})$.

ДокАЗАтЕльство. Утверждение (i) может быть найдено в [30; 1 1]. Для доказательства (ii) заметим, что $W \sim 2 M+f^{*} G$ для некоторого дивизора $G$ на $Z$. Так как $K_{\mathbb{P}(\mathscr{E})}=-3 M+f^{*}\left(c_{1}(\mathscr{E})+K_{Z}\right)$, то по формуле присоединения $G \sim$ $-c_{1}(\mathscr{E})-K_{Z}$. Наконец, (iii) следует из (ii) и того, что $-K_{W}=\left.M\right|_{W}$.

Мы можем считать, что дивизор $-K_{V}$ очень обилен (см. [6]). Тогда линейная система $\left|-K_{W}\right|=\left.|M|\right|_{W}$ задает морфизм $W \rightarrow \mathbb{P}^{g+1}$, отображающий слои $f$ в коники в $\mathbb{P}^{g+1}$. Пусть $\mathbb{P}_{z}^{2}-$ слой $\mathbb{P}(\mathscr{E}) \rightarrow Z$, и пусть $W_{z}:=\mathbb{P}_{z}^{2} \cap W$. Так как линейная система $\left.\left|-K_{W}\right|\right|_{W_{z}}$ задает вложение, то $\mid M \|_{\mathbb{P}_{z}^{2}}$ также задает вложение. Следовательно, линейная система $|M|$ не имеет базисных точек. Это означает, что расслоение $\mathscr{E}$ порождается глобальными сечениями. В частности, класс $c_{1}(\mathscr{E})=$ $-3 K_{Z}-\Delta$ численно эффективен и $c_{2}(\mathscr{E}) \geqslant 0$. Таким образом,

$$
-K_{W}^{3} \leqslant\left(-3 K_{Z}-\Delta\right) \cdot\left(-4 K_{Z}-\Delta\right)=12 K_{Z}^{2}+7 K_{Z} \cdot \Delta+\Delta^{2} .
$$

По лемме $5.3 p_{a}(\Delta) \geqslant 1$. Теперь наше утверждение вытекает из следующего.

ВСПОМОГАТЕЛЬНАЯ ЛЕММА 5.6. Пусть $Z$ - неособая поверхность почти дель Пецио, и пусть $\Delta$ - приведенная кривая на $Z$ такая, что $p_{a}(\Delta) \geqslant 1$ и дивизор $-3 K_{Z}-\Delta$ численно әффективен. Тогда

$$
12 K_{Z}^{2}+7 K_{Z} \cdot \Delta+\Delta^{2} \leqslant 54
$$

ДокАЗАтЕльство. Пусть $\sigma: Z \rightarrow Z^{\prime}-$ стягивание $(-1)$-кривой $L$. Положим $\Delta^{\prime}:=\sigma(\Delta)$. Тогда $K_{Z}=\sigma^{*} K_{Z^{\prime}}+L$ и $\Delta=\sigma^{*} \Delta^{\prime}-a L$, где $a \geqslant 0$. Следовательно,

$$
\begin{aligned}
& 2 p_{a}(\Delta)-2=\left(K_{Z}+\Delta\right) \cdot \Delta=\left(K_{Z^{\prime}}+\Delta^{\prime}\right) \cdot \Delta^{\prime}+(a-1) a L^{2} \leqslant 2 p_{a}\left(\Delta^{\prime}\right)-2, \\
& 12 K_{Z}^{2}+7 K_{Z} \cdot \Delta+\Delta^{2} \\
& \quad=12\left(\sigma^{*} K_{Z^{\prime}}+L\right)^{2}+7\left(\sigma^{*} K_{Z^{\prime}}+L\right) \cdot\left(\sigma^{*} \Delta^{\prime}-a L\right)+\left(\sigma^{*} \Delta^{\prime}-a L\right)^{2} \\
& \quad=12 K_{Z^{\prime}}^{2}-12+7 K_{Z^{\prime}} \cdot \Delta^{\prime}+7 a+\left(\Delta^{\prime}\right)^{2}-a^{2} \leqslant 12 K_{Z^{\prime}}^{2}+7 K_{Z^{\prime}} \cdot \Delta^{\prime}+\left(\Delta^{\prime}\right)^{2} .
\end{aligned}
$$

Таким образом, мы можем предполагать, что поверхность $Z$ не содержит $(-1)$-кривых, т.е. $Z$ изоморфна $\mathbb{P}^{2}$ или $\mathbb{F}_{n}$ с $n=0,2$. 
Рассмотрим случай $Z \simeq \mathbb{P}^{2}$. Пусть $d:=\operatorname{deg} \Delta$. Так как дивизор $-3 K_{Z}-\Delta$ численно эффективен, то $3 \leqslant d \leqslant 9$. Отсюда

$$
12 K_{Z}^{2}+7 K_{Z} \cdot \Delta+\Delta^{2}=108-21 d+d^{2} \leqslant 54 .
$$

Теперь рассмотрим случай $Z \simeq \mathbb{F}_{n}, n=0,2$. Пусть $\Sigma$ - минимальное сечение, и пусть $l-$ слой. Можно записать $\Delta \sim \alpha \Sigma+\beta l$ для некоторого $\alpha, \beta \in \mathbb{Z}$. Так как $p_{a}(\Delta) \geqslant 1$, то

$0 \leqslant\left(K_{\mathbb{F}_{n}}+\Delta\right) \cdot \Delta=-n \alpha(\alpha-2)+(\alpha-2) \beta+\alpha(\beta-2-n)=(\alpha-1)(-n \alpha+2 \beta-2)-2$.

$\mathrm{B}$ частности, $\alpha \geqslant 2$. Это также дает нам $-n \alpha+2 \beta-2 \geqslant 1,2 \beta \geqslant 3+n \alpha$. Далее,

$$
\begin{aligned}
12 K_{Z}^{2}+7 K_{Z} \cdot \Delta+\Delta^{2} & =96+14 n \alpha-14 \beta-7 n \alpha-14 \alpha-n \alpha^{2}+2 \alpha \beta \\
& =(7-\alpha)(n \alpha-2 \beta+14)-2 .
\end{aligned}
$$

Так как дивизор

$$
-3 K_{Z}-\Delta \sim(3-\alpha) \Sigma+(6+3 n-\beta) l
$$

численно эффективен, то $\alpha \leqslant 6$. Поэтому

$$
(7-\alpha)(n \alpha-2 \beta+14)-2 \leqslant 11(7-\alpha)-2 \leqslant 53 .
$$

ПреДПоложения 5.7. Докажем (iii) из предложения 5.2. Дополнительно к предположениям 5.1 мы теперь предположим, что $f: W \rightarrow Z-\mathbb{P}^{1}$-расслоение (в частности, $W$ неособо). Тогда $W=\mathbb{P}(\mathscr{E})$ для некоторого векторного расслоения $\mathscr{E}$ ранга 2 на $Z$. Пусть $L$ - тавтологический дивизор.

Из относительной точной последовательности Эйлера получаем

$$
-K_{W}=2 L+f^{*}\left(-K_{Z}-c_{1}\right) .
$$

Формула Хирша дает нам, что

$$
L^{2} \equiv L \cdot f^{*} c_{1}-f^{*} c_{2}
$$

Отсюда $L^{3}=c_{1}^{2}-c_{2}$ и

$$
-K_{W}^{3}=6 K_{Z}^{2}+2 c_{1}^{2}-8 c_{2} .
$$

По теореме Римана-Роха и двойственности Серра

$$
h^{0}(\mathscr{E})+h^{0}\left(\mathscr{E} \otimes \operatorname{det} \mathscr{E}^{*} \otimes \omega_{Z}\right) \geqslant \frac{1}{2}\left(c_{1}^{2}-2 c_{2}-K_{Z} \cdot c_{1}\right)+2
$$


Случай $Z=\mathbb{P}^{2}$. Обозначим $G:=f^{*} h$, где $h-$ прямая на $Z=\mathbb{P}^{2}$. Если $c_{1}$ четно, то мы можем считать, что $c_{1}=-2$. Тогда $L^{2} \cdot G=-2$ и $-K_{W}^{3}=62-8 c_{2}$. Предполагая $-K_{W}^{3} \geqslant 64$, мы получим $c_{2} \leqslant-1$. Из (5.8) имеем $h^{0}(\mathscr{E}) \geqslant 1-c_{2} \geqslant 2$, т.е. $\operatorname{dim}|L| \geqslant 1$. Так как $L^{2} \cdot G<0$, то линейная система $|L|$ имеет неподвижные компоненты. С другой стороны, $\operatorname{Pic}(W)=\mathbb{Z} \cdot M \oplus \mathbb{Z} \cdot G$. Следовательно, $|L-G| \neq \varnothing$. Но тогда

$$
0 \leqslant\left(-K_{W}\right) \cdot(L-G) \cdot G=(2 L+5 G) \cdot(L-G) \cdot G=2 L^{2} \cdot G+3 L \cdot G^{2}=-1
$$

\section{Противоречие.}

Пусть $c_{1}(\mathscr{E})$ нечетно. Тогда антиканонический дивизор $-K_{W}$ делится на 2 : $-K_{W}=2 D$, где $D=L+\frac{1}{2} f^{*}\left(-K_{Z}-c_{1}(\mathscr{E})\right)$. В этом случае общий элемент $D \in|D|$ является поверхностью почти дель Пещю с дювалевскими особенностями (см. [18]). Тогда $-K_{W}^{3}=8 K_{D}^{2}$. Предполагая $-K_{W}^{3}>64$, мы получим $K_{D}^{2}=9, D \simeq \mathbb{P}^{2}$ и $D_{D}=-K_{D}$. Заметим, что ограничение $H^{0}\left(W, \mathscr{O}_{W}(D)\right) \rightarrow$ $H^{0}\left(D, \mathscr{O}_{D}(D)\right)$ сюръективно. Следовательно, дивизор $D$ очень обилен и определяет вложение $V=V_{9} \hookrightarrow \mathbb{P}^{10}$ так, что слои $f$ отображаются в прямые в $\mathbb{P}^{10}$. Заметим, что $\phi: W \rightarrow V$ - экстремальное стягивание. Если $\phi$ имеет тип $(1,0)^{0}$, то $V$ имеет лишь терминальные горенштейновы особенности. В этом случае по теореме 1.3 имеем $-K_{W}^{3} \leqslant 64$. Предположим, что $\phi: W \rightarrow V$ дивизориально, и пусть $E-$ исключительный дивизор. Тогда $V \mathbb{Q}$-факториально и $\rho(V)=1$. Так как $D \simeq \mathbb{P}^{2}$, то $D$ не пересекает слоев $\phi$, т.е. $\phi(D) \cap \phi(E)=\varnothing$. Это возможно, только если $\phi(E)$ - точка. Следовательно, $V$ - конус над $\phi(D)$. Иначе говоря, $V \simeq \mathbb{P}(3,1,1,1)$. Мы получаем случай $(*)$ из предложения 5.2 .

Далее мы рассмотрим случай $Z \simeq \mathbb{F}_{n}$, где $n=0$ или 2 . Пусть $\Sigma$ и $l$-минимальное сечение и слой $\mathbb{F}_{n}$ соответственно. Мы можем предположить, что $c_{1}=c_{1}(\mathscr{E})=$ $a \Sigma+b l$ и $c_{2}=c_{2}(\mathscr{E})=c$. Если оба числа $a$ и $b$ четны, то мы можем считать, что $c_{1}=0$. Из (5.5) получаем

$$
-K_{W}=2 L-f^{*} K_{Z}=2 D
$$

где $D \sim L+f^{*}(\Sigma+(1+n / 2) l)$. Как и выше, общий элемент $D \in|D|$ изоморфен $\mathbb{P}^{2}$. С другой стороны, не сушествует доминантных морфизмов из $\mathbb{P}^{2}$ на $\mathbb{F}_{n}$. Противоречие.

Случай $Z \simeq \mathbb{P}^{1} \times \mathbb{P}^{1}$. В этом случае мы можем переставлять $a$ и $b$.

Случай, когда $a$ нечетно, $b$ четно. Мы можем считать, что $c_{1}=-3 \Sigma$. Из соотношений (5.5)-(5.7) получаем

$$
L^{2} \cdot f^{*} \Sigma=0, \quad L^{2} \cdot f^{*} l=-3, \quad-K_{W}=2 L+f^{*}(5 \Sigma+2 l), \quad-K_{W}^{3}=48-8 c .
$$

Отсюда $c \leqslant-2$. Из (5.8) получаем

$$
h^{0}(\mathscr{E})+h^{0}(\mathscr{E} \otimes \mathscr{O}(\Sigma-2 l)) \geqslant \frac{1}{2}\left(-2 c+3 K_{Z} \cdot \Sigma\right)+2=-c-1>0
$$


Следовательно, имеет место одно из двух: $|L| \neq \varnothing$ или $\left|L+f^{*} \Sigma-2 f^{*} l\right| \neq \varnothing$. С другой стороны,

$$
\begin{aligned}
& 0 \leqslant-K_{W} \cdot L \cdot f^{*} l=\left(2 L+f^{*}(5 \Sigma+2 l)\right) \cdot L \cdot f^{*} l=-1, \\
& 0 \leqslant-K_{W} \cdot\left(L+f^{*} \Sigma-2 f^{*} l\right) \cdot f^{*} \Sigma<0 .
\end{aligned}
$$

Противоречие.

Случай $c_{1}=-\Sigma-l$. Как и выше,

$$
\begin{gathered}
L^{2} \cdot f^{*} \Sigma=L^{2} \cdot f^{*} l=-1, \\
-K_{W}=2 L+3 f^{*}(\Sigma+l), \quad-K_{W}^{3}=\left(2 L+f^{*}(3 \Sigma+3 l)\right)^{3}=52-8 c .
\end{gathered}
$$

Это дает нам $c \leqslant-2$. Из (5.8) получаем

$$
h^{0}(\mathscr{E})+h^{0}(\mathscr{E} \otimes \mathscr{O}(-\Sigma-l)) \geqslant 1-c>0 .
$$

Следовательно, $|L| \neq \varnothing$. Так как $L^{2} \cdot f^{*} \Sigma=L^{2} \cdot f^{*} l=-1$, то $|L|$ имеет неподвижные компоненты. Запишем $L=F+|M|$, где $F$ - неподвижная часть. Тогда $M=f^{*} Q$, где $Q \sim \alpha \Sigma+\beta l, \alpha, \beta \geqslant 0$. Это дает нам, что

$$
0 \leqslant F \cdot\left(-K_{W}\right) \cdot f^{*} l=\left(L-f^{*}(\alpha \Sigma+\beta l)\right) \cdot\left(2 L+3 f^{*}(\Sigma+l)\right) \cdot f^{*} l=1-2 \alpha .
$$

Отсюда $\alpha=0$. Аналогично получаем $\beta=0$ и $F=0$. Противоречие.

Случай $Z \simeq \mathbb{F}_{2}$.

Случай $c_{1}=-\Sigma$. Тогда

$$
\begin{gathered}
L^{2} \cdot f^{*} \Sigma=2, \quad L^{2} \cdot f^{*} l=-1, \\
-K_{W}=2 L+f^{*}(3 \Sigma+4 l), \quad-K_{W}^{3}=\left(2 L+f^{*}(3 \Sigma+4 l)\right)^{3}=44-8 c_{2} .
\end{gathered}
$$

Отсюда $c_{2} \leqslant-3$. Согласно (5.8) имеем

$$
h^{0}(\mathscr{E})+h^{0}(\mathscr{E} \otimes \mathscr{O}(-\Sigma-4 l)) \geqslant 1-c_{2} \geqslant 4 .
$$

Поэтому $\operatorname{dim}|L| \geqslant 2$ (поскольку $\operatorname{dim}|L|>\operatorname{dim}\left|L-f^{*}(\Sigma+4 l)\right|$ ). Так как $L^{2} \cdot f^{*} l<0$, то линейная система $|L|$ имеет неподвижные компоненты. Запишем $|L|=F+|M|$, где $F$ - неподвижная, а $|M|$ - подвижная часть. Тогда

$$
-K_{W} \cdot(F+M) \cdot f^{*} l=-K_{W} \cdot L \cdot f^{*} l=1 .
$$

Отсюда $-K_{W} \cdot M \cdot f^{*} l \leqslant 1$ и $-K_{W} \cdot F \cdot f^{*} l \leqslant 1$. Так как $-K_{W} \cdot f^{*} \Sigma \cdot f^{*} l=2$, то дивизор $f^{*} \Sigma$ не может быть неподвижной компонентой $|L|$. Следовательно, $F$ имеет 
ровно одну компоненту, которая должна быть горизонтальной, и $M \sim f^{*}(\alpha \Sigma+\beta l)$ для некоторых $\alpha, \beta \geqslant 0$. Тогда

$$
-K_{W} \cdot M \cdot f^{*} l=-K_{W} \cdot f^{*}(\alpha \Sigma+\beta l) \cdot f^{*} l=2 \alpha .
$$

Таким образом, $\alpha=0$. Но тогда

$$
-K_{W} \cdot F \cdot f^{*} \Sigma=-K_{W} \cdot\left(L-\beta f^{*} l\right) \cdot f^{*} \Sigma=2-2 \beta .
$$

Единственная возможность: $\beta=1$. В этом случае $\operatorname{dim}|L|=\operatorname{dim}|M|=\operatorname{dim}|l|=1$. Противоречие.

$$
\begin{aligned}
& \text { Случай } c_{1}=-l \text {. Тогда } \\
& \qquad L^{2} \cdot f^{*} \Sigma=-1, \quad L^{2} \cdot f^{*} l=0, \\
& -K_{W}=2 L+f^{*}(2 \Sigma+5 l), \quad-K_{W}^{3}=\left(2 L+f^{*}(3 \Sigma+5 l)\right)^{3}=48-8 c_{2} .
\end{aligned}
$$

Таким образом, $c_{2} \leqslant-3$. Согласно (5.8) имеем

$$
h^{0}(\mathscr{E})+h^{0}(\mathscr{E} \otimes \mathscr{O}(-2 \Sigma-3 l)) \geqslant-c_{2}+1 \geqslant 4 .
$$

Отсюда $\operatorname{dim}|L| \geqslant 1$. Так как

$$
-K_{W} \cdot L \cdot f^{*} \Sigma=\left(2 L+f^{*}(2 \Sigma+5 l)\right) \cdot L \cdot f^{*} \Sigma=-1<0,
$$

то $f^{*} \Sigma$ - неподвижная компонента $|L|$. Далее,

$$
-K_{W} \cdot\left(L-f^{*} \Sigma\right) \cdot f^{*} l=\left(2 L+f^{*}(2 \Sigma+5 l)\right) \cdot\left(L-f^{*} \Sigma\right) \cdot f^{*} l=0 .
$$

Это противоречит тому, что $-K_{W}$ численно эффективен и объемен.

Случай $c_{1}=-\Sigma-l$. Тогда

$$
\begin{gathered}
L^{2} \cdot f^{*} \Sigma=1, \quad L^{2} \cdot f^{*} l=-1, \\
-K_{W}=2 L+f^{*}(3 \Sigma+5 l), \quad-K_{W}^{3}=\left(2 L+f^{*}(3 \Sigma+5 l)\right)^{3}=48-8 c_{2} .
\end{gathered}
$$

Отсюда $c_{2} \leqslant-3$. Из (5.8) получаем

$$
h^{0}(\mathscr{E})+h^{0}(\mathscr{E} \otimes \mathscr{O}(-\Sigma-3 l)) \geqslant 1-c_{2} \geqslant 4 .
$$

Таким образом, $\operatorname{dim}|L| \geqslant 1$. Так как $L^{2} \cdot f^{*} l=-1$, то линейная система $|L|$ имеет неподвижные компоненты. Запишем $|L|=F+|M|$, где $F$ - неподвижная, а $|M|-$ подвижная часть. Тогда

$$
-K_{W} \cdot(F+M) \cdot f^{*} l=-K_{W} \cdot L \cdot f^{*} l=1,
$$

поэтому $-K_{W} \cdot M \cdot f^{*} l \leqslant 1$ и $-K_{W} \cdot F \cdot f^{*} l \leqslant 1$. Так как $-K_{W} \cdot f^{*} \Sigma \cdot f^{*} l=$ 2 , то дивизор $f^{*} \Sigma$ не может быть неподвижной компонентой $|L|$. Следовательно, $F$ имеет ровно одну компоненту, которая должна быть горизонтальной и $M \sim$ $f^{*}(\alpha \Sigma+\beta l)$ для некоторых $\alpha, \beta \geqslant 0$. Тогда

$$
-K_{W} \cdot M \cdot f^{*} l=-K_{W} \cdot f^{*}(\alpha \Sigma+\beta l) \cdot f^{*} l=2 \alpha .
$$

Таким образом, $\alpha=0$. Отсюда получаем

$$
-K_{W} \cdot F \cdot f^{*} \Sigma=-K_{W} \cdot\left(L-\beta f^{*} l\right) \cdot f^{*} \Sigma=1-2 \beta<0 .
$$

\section{Противоречие.}

Теперь мы рассмотрим случай, когда $Z$ содержит $(-1)$-кривую. 
Лемма 5.8. Если $Z$ содержит (-1)-кривую, то существует многообразие почти Фано $W^{\prime}$, удовлетворяющее предположсениям (5.1), (5.7) и такое, что $-K_{W^{\prime}}^{3} \geqslant-K_{W}^{3}+6$. Более того, если $-K_{W^{\prime}}^{3}=-K_{W}^{3}+6$, то на $W^{\prime}$ линейная система $\left|-n K_{W^{\prime}}\right|$ может стягивать только $f^{\prime}$-вертикальнье дивизоры.

ДокАЗАТЕЛЬСТво. Пусть $C \subset Z-(-1)$-кривая, $\delta: Z \rightarrow Z^{\prime}$ - ее стягивание и $D:=f^{-1}(C)$. Тогда $D \simeq \mathbb{F}_{n}$ для некоторого $n$. Согласно лемме 5.4 для минимального сечения $\Sigma$ поверхности $D \simeq \mathbb{F}_{n}$ имеем $0 \geqslant K_{W} \cdot \Sigma=n-1$. Отсюда $n \leqslant 1$. Если $n=1$, то снова по лемме 5.4 существует коммутативная диаграмма

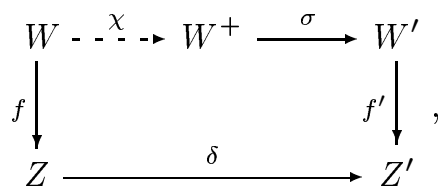

где $\chi$ - флоп, $\sigma$ - экстремальное дивизориальное стягивание дивизора в неособую точку и $f^{\prime}-\mathbb{P}^{1}$-расслоение. Тогда $W^{+}$снова является многообразием почти $\Phi$ ано, удовлетворяющим условиям 5.1 и 5.7. Так как $\sigma$ стягивает дивизор в точку, то же верно и для $W^{\prime}$. Ясно, что $-K_{W}^{3}=-K_{W^{+}}^{3}=-K_{W^{\prime}}^{3}-8$.

Случай $n=0$ разбирается аналогичным способом с использованием утверждения (iii) из леммы 5.4. Здесь $\chi=\mathrm{id}$ и $\sigma$ стягивает дивизор $S$ на слой морфизма $f^{\prime}$. Следовательно, $-K_{W}^{3}=-K_{W^{\prime}}^{3}-6$. Более того, если $\sigma(S)$ пересекает $K_{W^{\prime}}$-тривиальную кривую $C^{\prime}$, то для ее собственного прообраза $C \subset W$ имеем

$$
K_{W} \cdot C=\sigma^{*} K_{W^{\prime}} \cdot C+S \cdot C>K_{W^{\prime}} \cdot C^{\prime}=0 .
$$

Противоречие.

Закончим доказательство предложения 5.2 в случае, когда $Z$ содержит $(-1)$-кривую. По предположению индукции мы можем считать, что $-K_{W^{\prime}}^{3} \leqslant 72$. Если $-K_{W}^{3} \geqslant 66$, то $-K_{W^{\prime}}^{3}=72$ и $W^{\prime}$ - такое, как в $(*)$ в предложении 5.2 . Но это противоречит второму утверждению из леммы 5.8. Доказательство предложения 5.2 закончено.

\section{§6. Конструкция бирациональной перестройки}

ПРЕДПОЛОЖЕНИЯ 6.1. Как и выше, пусть $W$ - многообразие почти Фано, имеюшее лиш терминальные факториальные особенности. Пусть $\phi: W \rightarrow V-$ морфизм, заданный линейной системой $\left|-n K_{W}\right|$ для $n \gg 0$. Предположим, что $-K_{W}^{3} \geqslant 72$. Мы можем считать, что

(1) $W$ не имеет экстремальных лучей типа $(3,1)$ и $(3,2)$ (см. предложения 4.9 и 5.2 );

(2) $W$ не имеет экстремальных лучей типа $(2,0)^{-}$и $(2,1)^{-}$(см. предложение 4.5). 
Так как $W$ - многообразие почти Фано, то на $W$ должен существовать по крайней мере один $K$-отрицательньй экстремальный луч $R$. Согласно сказанному выше $R$ имеет тип $(2,1)_{\circ 1}^{-},(2,1)_{\circ 0}^{-}$или $(2,0)_{\circ}^{-}$.

Учитьвая следствие 4.7 , получим, что для многообразия $V$ имеет место по крайней мере один из следующих случаев:

(А) $V$ особо вдоль прямой $\Gamma$,

(В) $V$ содержит плоскость П.

Для дальнейшего удобно также выделить следующий случай:

(0) многообразие $V$ имеет по крайней мере одну особую точку $P$, не являюшуюся cDV.

Напомним, что трехмерная особенность назьвается cDV, если она гиперповерхностная и локально, с точностью до аналитической замены координат, задается уравнением $\phi(x, y, z)+t \psi(x, y, z, t)=0$, где $\phi(x, y, z)=0$ - уравнение (двумерной) дювалевской особенности [24].

Теперь наша теорема вытекает из следующего предложения.

ПРЕДЛОЖЕНИЕ 6.2. Пусть $V$ - многообразие Фано, имеющее лишь канонические горенштейновы особенности. Предположим, что $V$ удовлетворяет условиям (0), (A) или (В). Тогда $-K_{V}^{3} \leqslant 72$. Более того, если имеет место равенство $-K_{V}^{3}=72$, то многообразие $V$ изоморфно $\mathbb{P}(3,1,1,1)$ или $\mathbb{P}(6,4,1,1)$.

Если $-K_{V}^{3}>54$, то согласно [6] (см. $\left.\S 2\right)$ антиканоническая линейная система задает вложение $V=V_{2 g-2} \subset \mathbb{P}^{g+1}$. Более того, его образ $V_{2 g-2} \subset \mathbb{P}^{g+1}$ является пересечением квадрик [6; лемма 3]. Положим $\mathscr{L}:=\left|-K_{V}\right|$. Тогда $\operatorname{dim} \mathscr{L}=g+1>38$. Рассмотрим следуюшую подсистему $\mathscr{H} \subset \mathscr{L}:$

$$
\begin{array}{ll}
\mathscr{H}:=\{H \in \mathscr{L} \mid H \ni P\} & \text { в случае (0); } \\
\mathscr{H}:=\{H \in \mathscr{L} \mid H \supset \Gamma\} & \text { в случае (А); } \\
\mathscr{H}:=\{H \mid H+\Pi \in \mathscr{L}\} & \text { в случае (В). }
\end{array}
$$

Ясно, что

$$
\operatorname{dim} \mathscr{H}=\left\{\begin{array}{l}
\operatorname{dim} \mathscr{L}-1 \text { в случае }(0), \\
\operatorname{dim} \mathscr{L}-2 \text { в случае }(\mathrm{A}), \\
\operatorname{dim} \mathscr{L}-3 \text { в случае }(\mathrm{B}) .
\end{array}\right.
$$

Пусть теперь $\mathscr{L}_{W}$ и $\mathscr{H}_{W}$ - собственные прообразы на $W$ линейных систем $\mathscr{L}$ и $\mathscr{H}$ соответственно.

В случае (0) согласно [24] существует по крайней мере один исключительный дивизор $B_{i}$ с центром в $P$ и дискрепантностью $a\left(B_{i}\right)=0$. Запишем $\phi^{*} \mathscr{H}_{W}=\mathscr{H}+$ $B$, где $B=\sum b_{i} B_{i}-$ ненулевой эффективный (цельй) исключительньй дивизор над $P$. Таким образом,

$$
K_{W}+\mathscr{H}_{W}+B=\phi^{*}\left(K_{V}+\mathscr{H}\right)
$$


Аналогично во всех случаях имеем

$$
\begin{aligned}
& \left.K_{W}+\mathscr{H}_{W}+B=\phi^{*}\left(K_{V}+\mathscr{H}\right) \sim 0 \quad \text { (случаи }(0) \text { и }(\mathrm{A})\right), \\
& \left.K_{W}+\mathscr{H}_{W}+B=\phi^{*}\left(K_{V}+\mathscr{H}+\Pi\right) \sim 0 \quad \text { (случай }(\mathrm{B})\right),
\end{aligned}
$$

где $B$ - целый эффективный ненулевой дивизор.

ЛЕмма 6.3. Образ многообразия $V$ при отображении $\Phi_{\mathscr{H}}$, заданном линейной системой $\mathscr{H}$, трехмерен.

ДокАЗАТЕЛЬство. Отметим, что в случае (0) отображение $\Phi_{\mathscr{H}}-$ не что иное, как проекция из точки $P$. Предположим, что $\operatorname{dim} \Phi_{\mathscr{H}}(V) \leqslant 2$. Тогда $V-$ конус над $\Phi_{\mathscr{H}}(V)$ с вершиной в $P$. Но в этом случае особенность $P \in X$ не может быть канонической (см., например, [24; 2.14]).

Рассмотрим теперь случай $(\mathrm{A})$ (случай (В) рассматривается аналогично). Как и выше, заметим, что $\Phi_{\mathscr{H}}-$ проекция из прямой Г. Если $\Phi_{\mathscr{H}}(V)-$ кривая $C$, то многообразие $V$ является конусом над $C$ с вершиной в $Г$. Но тогда общий элемент $L \in \mathscr{L}$ также является конусом с вершиной в $L \cap \Gamma$. Это противоречит тому, что $L-$ поверхность типа К3 с дювалевскими особенностями. Пусть теперь $\Phi_{\mathscr{H}}(V)$ - поверхность. Тогда общий слой отображения $\Phi_{\mathscr{H}}: V \rightarrow \Phi_{\mathscr{H}}(V)$ одномерен. С другой стороны, слои этого отображения высекаются на $V$ плоскостями $\Lambda$, проходящими через Г. Каждая такая плоскость высекает на $V$ схему, являющуюся пересечением квадрик. Следовательно, $\Lambda \cap V=\Gamma+\Gamma_{\Lambda}$, где $\Gamma_{\Lambda}-$ прямая, причем слои $\Phi_{\mathscr{H}}-$ это такие прямые $\Gamma_{\Lambda}$. Таким образом, через общую точку на $V$ проходит прямая. В этом случае несложно получить оценку $-K_{V}^{3} \leqslant 46$ (см. [6; лемма 5]).

Из теоремы 2.1 и из обращения присоединения $[22 ; 3.3,9.5]$ немедленно получаем

СлЕДСТВИЕ 6.4. Пара $\left(V,\left|-K_{V}\right|\right)$ имеет лишь канонические особенности.

Мы можем записать

$$
K_{W}+\mathscr{L}_{W}=\phi^{*}\left(K_{V}+\mathscr{L}\right) \sim 0
$$

поэтому пара $\left(W, \mathscr{L}_{W}\right)$ канонична.

ЗАмЕчАниЕ 6.5. Согласно [24; следствие 2.14] все компоненты дивизора $B$ бирационально линейчатые поверхности.

ЛЕмма 6.6. В обозначениях 6.1 мы можем въбрать модификацию ф так, ч

(i) пара $\left(W, \mathscr{H}_{W}\right)$ канонична;

(ii) линейная система $\mathscr{H}_{W}$ численно әффективна. 
ДоказАтельство. Рассмотрим сначала случай (0). Согласно [24; теорема 2.11] существует раздутие $\phi_{1}: V_{1} \rightarrow V$ такое, что

a) многообразие $V_{1}$ нормально;

b) собственный прообраз $H_{1}$ обшего дивизора $H \in \mathscr{H}$ является дивизором Картье и имеет лишь дювалевские особенности;

c) $K_{V_{1}}=\phi_{1}^{*} K_{V}$ (в частности, $K_{V_{1}}$ - дивизор Картье и многообразие $V_{1}$ имеет лишш канонические особенности).

По обращению присоединения $[22 ; 3.3,9.5]$ пара $\left(V_{1}, \mathscr{H}_{1}\right)$ чисто логтерминальна. Так как $\mathscr{H}_{1}$ составлена из дивизоров Картье, то пара $\left(V_{1}, \mathscr{H}_{1}\right)$ канонична.

Рассмотрим терминальную $\mathbb{Q}$-факториальную модификацию $g_{1}: W \rightarrow V_{1}$ многообразия $V_{1}$. Композиция

$$
\phi: W \stackrel{g_{1}}{\longrightarrow} V_{1} \stackrel{\phi_{1}}{\longrightarrow} V
$$

является терминальной $\mathbb{Q}$-факториальной модификацией для $V$. В этом случае $K_{W}=g_{1}^{*} K_{V_{1}}$ и $\mathscr{H}_{W}=g_{1}^{*} \mathscr{H}_{1}-E$, где $E \geqslant 0$. Таким образом, $K_{W}+\mathscr{H}_{W}=$ $g_{1}^{*}\left(K_{V_{1}}+\mathscr{H}_{1}\right)-E$. Так как пара $\left(V_{1}, \mathscr{H}_{1}\right)$ канонична, то $E=0$ и пара $\left(W, \mathscr{H}_{W}\right)$ также канонична. Для любой другой терминальной модификации $\phi^{\prime}: W^{\prime} \rightarrow V$ отображение $W \rightarrow W^{\prime}$ является изоморфизмом в коразмерности 1 и поэтому крепантно. По лемме 3.1 пара $\left(W^{\prime}, \mathscr{H}^{\prime}\right)$ также канонична.

Так как линейная система $\mathscr{H}_{W}$ не имеет неподвижных компонент, то существует лишь конечное число кривых $C_{i} \subset W$, отрицательно пересекающих $\mathscr{H}_{W}$, и эти кривые содержатся в Bs $\mathscr{H}_{W} \subset \phi^{-1}(P)$. Применим $\mathscr{H}_{W}$-программу минимальных моделей к $W$ над $V$. После конечного числа $\mathscr{H}_{W}$-флопов мы получим новую терминальную модификацию $\phi^{\prime}: W^{\prime} \rightarrow V$, на которой линейная система $\mathscr{H}_{W^{\prime}}$ численно эффективна. Заменяя $W$ на $W^{\prime}$, мы можем считать, что $\mathscr{H}_{W}$ численно эффективна.

В случаях (A) и (В) мы покажем, что линейная система $\mathscr{H}_{W}$ не имеет неподвижных компонент и базисных точек. В частности, пара $\left(W, \mathscr{H}_{W}\right)$ терминальна.

Рассмотрим случай (А). Так как $\mathscr{H}$ - линейная система гиперплоских сечений, проходящих через $\Gamma$, то для доказательства леммы достаточно только показать, что морфизм $\phi$ пропускается через раздутие $Г$ как приведенной подсхемы в $V$.

Поскольку каж дое крепантное раздутие может быть продолжено до терминальной $\mathbb{Q}$-факториальной модификации, то требуемое немедленно вытекает из следуюшего утверждения.

УТВЕРЖДЕНИЕ 6.7. Пусть $V$ - трехмерное многообразие с особенностями типа $c D V$, и пусть $\Gamma \subset \operatorname{Sing}(V)$ - одномерная неприводимая компонента. Предположим, что кривая $\Gamma$ неособа. Тогда раздутие $\sigma: \widetilde{V} \rightarrow V$ кривой $\Gamma$ как приведенной подсхемы крепантно. В частности, $\widetilde{V}$ нормально и имеет лишь особенности типа $c D V$.

ДокАЗАТЕЛЬСТво. Утверждение локальное. Поэтому мы можем считать, что $V$ - аналитическая окрестность некоторой точки $P \in \Gamma$. Таким образом, мы можем считать, что $V$ - гиперповерхностная особенность, заданная уравнением

$$
\psi_{0}(x, y, z)+t \psi_{1}(x, y, z)+t^{2} \psi_{2}(x, y, z)+\cdots=0
$$


где $\psi_{0}(x, y, z)=0$ - уравнение дювалевской особенности, а кривая $Г$ задана уравнениями $x=y=z=0$. Поскольку $V$ особо вдоль $\Gamma$, то mult $(0,0,0) \psi_{k} \geqslant 2$, а так как $V$ имеет лишь особенности типа $\mathrm{cDV}$, то $\operatorname{mult}_{(0,0,0)} \psi_{0}=2$. Тогда в карте $x \neq 0$ многообразие $\widetilde{V}$ задается уравнением

$$
\psi_{0}(x, y x, z x) x^{-2}+t \psi_{1}(x, y x, z x) x^{-2}+t^{2} \psi_{2}(x, y x, z x) x^{-2}+\cdots=0 .
$$

Здесь $\psi_{0}(x, y x, z x) x^{-2}$ не делится на $x$ и $\psi_{0}(x, y x, z x) x^{-2}=0$ - уравнение неособой или дювалевской точки. Это показывает, что $\widetilde{V}$ имеет лишь особенности типа cDV в нашей карте и слой над точкой $P$ одномерен. Из симметрии это же верно и в других картах. Следовательно, все компоненты исключительного дивизора сюръективно отображаются на Г. Из формулы присоединения тогда легко видеть, что морфизм $\sigma$ крепантен.

Случай (В) рассматривается аналогично. Лемма 6.6 доказана.

Применим теперь $\left(K_{W}+\mathscr{H}_{W}\right)$-программу минимальных моделей. На каждом шаге соотношение (6.1) сохраняется, поэтому логдивизор $K+\mathscr{H} \equiv-B$ не может быть численно эффективньм. В конце мы получим каноническую пару $\left(X, \mathscr{H}_{X}\right)$ и $\left(K_{X}+\mathscr{H}_{X}\right)$-отрицательное стягивание $f: X \rightarrow Z$ на многообразие меньшей размерности $Z$. Более того, многообразие $X$ имеет лишш терминальные $\mathbb{Q}$-факториальные особенности (однако $X$ необязательно горенштейново). По лемме 3.4 линейная система $\mathscr{H}_{X}$ численно эффективна и составлена из дивизоров Картье. По лемме 3.1 пара $\left(X, \mathscr{L}_{X}\right)$ канонична и $\mathscr{L}_{X} \subset\left|-K_{X}\right|$. Многообразие Фано $V=V_{2 g-2} \subset \mathbb{P}^{g+1}$ является образом $X$ при бирациональном отображении, заданном линейной системой $\mathscr{L}_{X}$. Согласно $(6.1)$ имеем

$$
K_{X}+\mathscr{H}_{X}+B_{X} \sim 0
$$

По лемме 6.3 образ $\Phi_{\mathscr{H}_{X}}(X)$ трехмерен. В частности, линейная система $\mathscr{H}_{X}$ не является прообразом линейной системы на $Z$, т.е. $\mathscr{H}_{X}$ обильна над $Z$.

Далее всюду мы предположим, что $-K_{V}^{3} \geqslant 72$. Тогда согласно (2.1) имеем $\operatorname{dim}\left|-K_{V}\right| \geqslant 38$ и $\operatorname{dim} \mathscr{H} \geqslant 35$. Отсюда

$$
\begin{gathered}
\operatorname{dim}\left|-K_{X}\right| \geqslant \operatorname{dim} \mathscr{L}_{X}=\operatorname{dim}\left|-K_{V}\right| \geqslant 38 \\
\operatorname{dim}|H| \geqslant \operatorname{dim} \mathscr{H} \geqslant 35,
\end{gathered}
$$

где $H \in \mathscr{H}_{X}-$ общий дивизор.

Для $Z$ имеются лишь следуюшие возможности:

a) $Z$ - точка,

б) $Z$-кривая,

в) $Z$ - поверхность.

В случае б) общий слой $X_{\eta}$ является неособой поверхностью дель Пеццо, причем дивизоры $\left.H\right|_{X_{\eta}}$ и $-\left(K_{X_{\eta}}+\left.H\right|_{X_{\eta}}\right)$ обильны. Отсюда $X_{\eta} \simeq \mathbb{P}^{2}$ или $\mathbb{P}^{1} \times \mathbb{P}^{1}$. Далее в следуюших параграфах мы рассмотрим случаи в зависимости от размерности многообразия $Z$ и типа слоя $X_{\eta}$. В каждом случае мы получим противоречие 
с (6.2), (6.3) или покажем, что в (6.2) имеют место равенства. В последнем случае $\left|-K_{X}\right|=\mathscr{L}_{X}$ и бирациональное отображение $X \rightarrow V$ задается полной линейной системой $\left|-K_{X}\right|$. Тогда будет несложно показать, что для $V$ имеются только две возможности из примера 1.4 .

\section{$\S 7$. Случай, когда $Z$ - точка}

ПРЕДПОЛОЖЕния 7.1. В этом параграфе мы рассмотрим случай, когда $Z-$ точка. Тогда $\rho(X)=1$ и $X$ - многообразие $\Phi$ ано с $\mathbb{Q}$-факториальными терминальными особенностями. В этой ситуации $\operatorname{Pic} X \simeq \mathbb{Z}$ (см., например, [33; предложение 2.1.2]. Пусть $G$ - обильный дивизор Картье, порождаюший Pic $X$. Мы можем записать $-K_{X} \equiv r G$ и $\mathscr{H}_{X} \sim a G$ для некоторых $r \in \mathbb{Q}, r>0$ и $a \in \mathbb{N}$. Такое $r$ называется индексом Фано $X$. Так как дивизор $-\left(K_{X}+\mathscr{H}_{X}\right)$ обилен, то $r>a \geqslant 1$.

ПРЕДЛОЖЕНИЕ 7.2. В обозначениях 7.1 имеем $\operatorname{dim}\left|-K_{X}\right| \leqslant 34$.

ДокАЗАТЕЛЬСТво. Если $X$ имеет лишш горенштейновы особенности, то по теореме 1.3 имеем $\operatorname{dim}\left|-K_{X}\right| \leqslant 34$. Следовательно, $X$ имеет по крайней мере одну точку индекса $>1$. Далее мы воспользуемся следуюшей теоремой.

Tеорема 7.3 (см. [34]). Пусть $X$ - трехмерное многообразие Фано с терминальными особенностями. Предположим, ито $X$ имеет по крайней мере одну точку индекса $>1$ и индекс Фано $r>1$. Тогда $X$ допускает одно из следующих вложений во взвешенное проективное пространство (используется нумерация из работы [34]):

[1]. $X=X_{6} \subset \mathbb{P}(1,1,2,3, i), \quad i=2,3,4,5,6$;

[2]. $X=X_{4} \subset \mathbb{P}(1,1,1,2, i), \quad i=2,3$;

[3]. $X=X_{3} \subset \mathbb{P}(1,1,1,1,2)$;

[5]. $X=\mathbb{P}(1,1,1,2), \quad r=5 / 2$.

Напомним, что взвешенное проективное пространство $\mathbb{P}=\mathbb{P}\left(w_{0}, \ldots, w_{n}\right)$ называется нормализованным, если $\operatorname{gcd}\left(w_{0}, \ldots, w_{j-1}, w_{j+1}, \ldots, w_{n}\right)=1$ для $j=0, \ldots, n$. При этом условии канонический дивизор вычисляется по формуле $\mathscr{O}_{\mathbb{P}}\left(K_{\mathbb{P}}\right)=\mathscr{O}_{\mathbb{P}}\left(-\sum d_{j}\right)$. Все взвешенные проективные пространства $\mathbb{P}$ в теореме 7.3 неособы в коразмерности 2. Поэтому в случаях [1]-[3] верна обычная формула присоединения $K_{X}=\left.\left(K_{\mathbb{P}}+X\right)\right|_{X}$. Из точной последовательности

$$
0 \longrightarrow \mathscr{O}_{\mathbb{P}}\left(-K_{\mathbb{P}}-2 X\right) \longrightarrow \mathscr{O}_{\mathbb{P}}\left(-K_{\mathbb{P}}-X\right) \longrightarrow \mathscr{O}_{X}\left(-K_{X}\right) \longrightarrow 0
$$

и обрашения в нуль $H^{1}\left(\mathbb{P}, \mathscr{O}_{\mathbb{P}}\left(-K_{\mathbb{P}}-2 X\right)\right)$ несложно вычислить размерность $\left|-K_{X}\right|$. Здесь используется тот факт, что $H^{0}(\mathbb{P}, \mathscr{O}(d))$ естественно изоморфно компоненте степени $d$ кольца многочленов $\mathbb{C}\left[x_{0}, \ldots, x_{n}\right]$, градуированного условиями $\operatorname{deg} x_{j}=$ $w_{j}$. Тогда в случаях [1]-[3] максимальное значение $\operatorname{dim}\left|-K_{X}\right|=30$ достигается в случае [1] при $i=6$. В случае [5] имеем $-K_{X} \sim \mathscr{O}(5)$ и $\operatorname{dim}\left|-K_{X}\right|=33$. 


\section{§8. Случай, когда $X_{\eta} \simeq \mathbb{P}^{2}$}

Так как $H^{1}\left(Z, \mathscr{O}_{Z}\right)=H^{1}\left(X, \mathscr{O}_{X}\right)=0$ (по теореме Каваматы-Фивега об обрашении в нуль), то $Z \simeq \mathbb{P}^{1}$. Пусть $H \in \mathscr{H}_{X}$ - общий дивизор. Дивизор $-\left(K_{X_{\eta}}+\left.H\right|_{X_{\eta}}\right)$ обилен, поэтому $\left.H\right|_{X_{\eta}} \simeq \mathscr{O}_{\mathbb{P}^{2}}(1)$ или $\mathscr{O}_{\mathbb{P}^{2}}(2)$.

Рассмотрим сначала случай $\left.H\right|_{X_{\eta}} \simeq \mathscr{O}_{\mathbb{P}^{2}}(1)$. Следующий факт хорошо известен (см., например, [35]). Для удобства читателя мы приведем его с доказательством.

ЛЕмма 8.1. Если $\left.H\right|_{X_{\eta}} \simeq \mathscr{O}_{\mathbb{P}^{2}}(1)$, то $f$ является $\mathbb{P}^{2}$-расслоением.

ДокАЗАТЕЛЬСтво. Пусть $S=g^{*} P$ - произвольный слой. Тогда $H^{2} \cdot S=$ $H^{2} \cdot X_{\eta}=1$. Следовательно, $S$ приведен и неприводим. Поскольку морфизм $f$ плоский, функция $\chi\left(X_{z}, \mathscr{O}_{X_{z}}(H)\right)$ локально постоянна. Таким образом, $\chi\left(S, \mathscr{O}_{S}(H)\right)=$ $\chi\left(\mathscr{O}_{\mathbb{P}^{2}}, \mathscr{O}_{\mathbb{P}^{2}}(1)\right)=3$. С другой стороны, $h^{2}\left(S, \mathscr{O}_{S}(H)\right)=h^{0}\left(S, \omega_{S} \otimes \mathscr{O}_{S}(-H)\right)=0$. Отсюда $h^{0}(S, \mathscr{O}(H)) \geqslant 3$. Напомним определение $\Delta$-рода поляризованного многообразия $(Y, \mathscr{M})($ см. [36]):

$$
\Delta(Y, \mathscr{M})=\operatorname{dim} Y+\mathscr{M}^{\operatorname{dim} Y}-h^{0}(Y, \mathscr{M})
$$

Известно, что $\Delta(Y, \mathscr{M}) \geqslant 0$ и в случае $\Delta(Y, \mathscr{M})=0$ многообразие $Y$ нормально, а пучок $\mathscr{M}$ является очень обильным [36]. В нашем случае имеем $\Delta\left(S, \mathscr{O}_{S}(H)\right) \leqslant 0$ и $\mathscr{O}_{S}(H)^{2}=1$. Отсюда $S \simeq \mathbb{P}^{2}$.

ПРЕДЛОЖЕНИЕ 8.2. Пусть $X \rightarrow Z=\mathbb{P}^{1}-\mathbb{P}^{2}$-расслоение. Предположим, что пара $\left(X,\left|-K_{X}\right|\right)$ канонична. Тогда $\operatorname{dim}\left|-K_{X}\right| \leqslant 38$. Более того, если $\operatorname{dim}\left|-K_{X}\right|=38$, mо $X=\mathbb{P}\left(\mathscr{O}_{\mathbb{P}^{1}}(6) \oplus \mathscr{O}_{\mathbb{P}^{1}}(2) \oplus \mathscr{O}_{\mathbb{P}^{1}}\right)$ и антиканонический образ $X$ - взвешенное проективное пространство $\mathbb{P}(6,4,1,1)$.

ДокаЗАТЕЛЬСТво. Мы можем считать, что $X=\mathbb{P}_{\mathbb{P}^{1}}(\mathscr{E})$, где

$$
\mathscr{E}=\mathscr{O}_{\mathbb{P}^{1}}\left(d_{1}\right) \oplus \mathscr{O}_{\mathbb{P}^{1}}\left(d_{2}\right) \oplus \mathscr{O}_{\mathbb{P}^{1}}\left(d_{3}\right), \quad d_{1} \geqslant d_{2} \geqslant d_{3}=0 .
$$

Обозначим через $F$ класс слоя $f$, а через $M$ - тавтологический дивизор, т.е. дивизор такой, что $\mathscr{O}_{X}(M) \simeq \mathscr{O}_{\mathbb{P}(\mathscr{E})}(1)$. Положим $d:=\sum d_{i}$. Относительная точная последовательность Эйлера дает нам

$$
-K_{X} \sim 3 M+(2-d) F
$$

Далее, пусть $\mathscr{E}^{\prime}:=\mathscr{O}_{\mathbb{P}^{1}}\left(d_{1}\right) \oplus \mathscr{O}_{\mathbb{P}^{1}}\left(d_{2}\right)$. Тогда

$$
\begin{aligned}
H^{0}(X, & \left.\mathscr{O}_{X}\left(-K_{X}\right)\right)=H^{0}\left(\mathbb{P}(\mathscr{E}), \mathscr{O}_{\mathbb{P}(\mathscr{E})}(3) \otimes f^{*} \mathscr{O}_{\mathbb{P}^{1}}(2-d)\right) \\
\simeq & H^{0}\left(\mathbb{P}^{1}, S^{3} \mathscr{E} \otimes \mathscr{O}_{\mathbb{P}^{1}}(2-d)\right) \\
= & H^{0}\left(\mathbb{P}^{1}, S^{3} \mathscr{E}^{\prime}(2-d)\right) \oplus H^{0}\left(\mathbb{P}^{1}, S^{2} \mathscr{E}^{\prime}(2-d)\right) \\
& \oplus H^{0}\left(\mathbb{P}^{1}, \mathscr{E}^{\prime}(2-d)\right) \oplus H^{0}\left(\mathbb{P}^{1}, \mathscr{O}_{\mathbb{P}^{1}}(2-d)\right),
\end{aligned}
$$


где

$$
H^{0}\left(\mathbb{P}^{1}, S^{m} \mathscr{E}^{\prime}(2-d)\right)=\bigoplus_{i=0}^{m} H^{0}\left(\mathbb{P}^{1}, \mathscr{O}_{\mathbb{P}^{1}}\left(i d_{1}+(m-i) d_{2}+2-d\right)\right) .
$$

С другой стороны, пара $\left(X,\left|-K_{X}\right|\right)$ канонична (так как $\left.\left|-K_{X}\right| \supset \mathscr{L}_{X}\right)$. В частности, линейная система $\left|-K_{X}\right|$ не имеет неподвижных компонент и ее общий элемент может иметь лишш изолированные особенности.

Ясно, что $H^{0}\left(X, M-d_{1} F\right) \simeq H^{0}\left(\mathbb{P}^{1}, \mathscr{E}\left(-d_{1}\right)\right) \neq 0$. Поэтому существует эффективный дивизор $D \sim M-d_{1} F$. Так как дивизор $M$ численно эффективен, то $-K_{X} \cdot D \cdot M \geqslant 0$. Вычисляя $-K_{X} \cdot D \cdot M$ с использованием формулы Хирша $M^{2}=d M \cdot F$, получим

$$
2 d_{2}+2-d_{1} \geqslant 0 .
$$

Если теперь $d_{2}=0$, то $d_{1}=d \leqslant 2$ и из (8.1) получаем $\operatorname{dim}\left|-K_{X}\right|=29$. Предположим, что $d_{2}>0$. Рассмотрим разложение $\mathscr{E}=\mathscr{E}^{\prime} \oplus \mathscr{O}_{\mathbb{P}^{1}}$, и пусть $C \subset X$ - сечение, соответствуюшее сюръекции $\mathscr{E} \rightarrow \mathscr{O}$. Общее сечение $L \in\left|-K_{X}\right|=|3 M+(2-d) F|$ имеет кратность $\leqslant 1$ в обшей точке $C$, а сама кривая $C$ задается обращением в нуль всех сечений $H^{0}\left(\mathbb{P}^{1}, \mathscr{E}^{\prime}\right) \subset H^{0}\left(\mathbb{P}^{1}, \mathscr{E}\right)$. Поэтому в сумме $(8.1)$ член $H^{0}\left(\mathbb{P}^{1}, \mathscr{E}^{\prime}(2-d)\right)$ ненулевой. Это значит, что $d_{1}+2-d \geqslant 0$. Таким образом, $d_{2} \leqslant 2$ и $d_{1} \leqslant 2 d_{2}+2 \leqslant 6$. Из этих неравенств получаем следующие возможности для $\left(d_{1}, d_{2}\right)$ :

$$
(1,1),(2,1),(2,2),(3,1),(3,2),(4,1),(4,2),(5,2),(6,2) .
$$

Из (8.1) немедленно вычисляем, что $\operatorname{dim}\left|-K_{X}\right| \leqslant 38$, причем равенство имеет место только при $\left(d_{1}, d_{2}\right)=(6,2)$. Последнее утверждение предложения 8.2 следует из конструкции Фано (см. [9; гл. 4, замечание 4.2]). Это доказывает предложение 8.2.

ПрЕДПоЛОЖЕНИя 8.3. Начиная с этого момента и до конца параграфа мы предполагаем, что $X_{\eta} \simeq \mathbb{P}^{2}$ и $\left.H\right|_{X_{\eta}} \simeq \mathscr{O}_{\mathbb{P}^{2}}(2)$.

Будет доказано следующее

ПРЕДЛОЖЕНИЕ 8.4. В обозначениях 8.3 существует $\left(K_{X}+\mathscr{L}\right)$-крепантное бирациональное отображсение $X \rightarrow X_{0}$ на $\mathbb{P}^{2}$-расслоение $X_{0}$ над $Z$.

Отсюда будет следовать теорема 1.5 в предположениях 8.3. Действительно, тогда по предложению 8.2 имеем $\operatorname{dim} \mathscr{L}=\operatorname{dim} \mathscr{L}_{X_{0}} \leqslant \operatorname{dim}\left|-K_{X_{0}}\right| \leqslant 38$ и, если имеют место равенства, то образы $X$ и $X_{0}$ при бирациональных отображениях, заданных линейньми системами $\mathscr{L}$ и $\left|-K_{X_{0}}\right|$, совпадают.

Лемма 8.5. Пусть $S=f^{*} z_{0}, z_{0} \in Z,-$ приведеннъй слой. Тогда

(i) $S$ - нормальная поверхность;

(ii) $S \simeq \mathbb{P}^{2}$ u.лu $\mathbb{W}_{4}$;

(iii) пара $(X, S)$ чисто логтерминальна.

ДокАЗАТЕльство. Как и в доказательстве леммы 8.1, получаем $h^{0}(S, \mathscr{O}(H)) \geqslant$ 6 и $\Delta\left(S, \mathscr{O}_{S}(H)\right)=0$. Поэтому поверхность $S$ нормальна и допускает вложение $S \hookrightarrow \mathbb{P}^{5}$ как поверхность степени 4 (так как $\mathscr{O}_{S}(H)^{2}=4$ ). Хорошо известно, что тогда для $S$ имеются только возможности п. (ii). Пункт (iii) следует теперь из обращения присоединения $[22 ; 3.3,9.5]$, поскольку $X$ неособо в коразмерности 2 . 
СлЕДСтвИЕ 8.6. Расслоение $f: X \rightarrow Z$ не имеет кратных слоев.

ДокАЗАТЕЛЬСтво. Предположим, что $f^{*} z_{0}=m S-$ слой кратности $m>1$. Рассмотрим $X$ как малую окрестность $S$ и рассмотрим замену базы

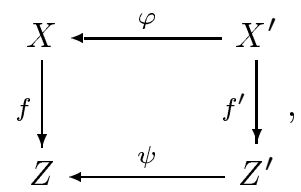

где $\psi$ - накрытие, локально заданное $t \rightarrow t^{m}$, а $X^{\prime}-$ нормализация доминантной компоненты $X \times{ }_{Z} Z^{\prime}$. Тогда $\varphi$ - конечное циклическое накрытие степени $m$, неразветвленное вне $\operatorname{Sing} X$. Пусть $S^{\prime}:=\varphi^{-1}(S)$. Тогда $S^{\prime}-$ дивизор Картье и является схемным слоем: $S^{\prime}=f^{\prime *} z_{0}^{\prime}$, где $z_{0}^{\prime}=\psi^{-1}\left(z_{0}\right)$. Так как $\operatorname{Sing} X-$ конечное множество, то компоненты $S^{\prime}$ могут пересекаться только в конечном множестве точек $\varphi^{-1}(\operatorname{Sing} X)$. Это противоречит лемме 8.7 ниже. Поэтому слой $S^{\prime}$ неприводим и приведен. По лемме $8.5 S^{\prime} \simeq \mathbb{P}^{2}$ или $\mathbb{W}_{4}$. Однако любая циклическая группа, действующая на $\mathbb{P}^{2}$ или $\mathbb{W}_{4}$, имеет на этой поверхности кривую неподвижных точек. Противоречие показывает, что $m=1$.

Лемма 8.7. Пусть $P \in Y$ - трехмерная терминальная особенность, и пусть $D^{\prime}, D^{\prime \prime}$ - эффективные дивизорь Вейля такие, что $D=D^{\prime}+D^{\prime \prime}$ является дивизором Картье. Тогда

$$
\operatorname{Supp} D^{\prime} \cap \operatorname{Supp} D^{\prime \prime} \geqslant 1 \text {. }
$$

ДоказАТЕльство. Предположим, что $\operatorname{Supp} D^{\prime} \cap \operatorname{Supp} D^{\prime \prime}=\{P\}$. Утверждение не изменится, если мы заменим особенность $P \in Y$ на ее каноническое накрытие. Таким образом, мы можем считать, что $P \in Y$ - изолированная гиперповерхностная особенность. Тогда $D$ - локально полное пересечение и $\operatorname{Supp} D^{\prime} \cap$ $\operatorname{Supp} D^{\prime \prime}=\{P\}$, что невозможно.

СлЕДСТВИЕ 8.8. Если $S \simeq \mathbb{P}^{2}$, то многообразие $X$ неособо вдоль $S$. Если $S \simeq \mathbb{W}_{4}$, то $X$ имеет ровно одну особую точку - вериину конуса $\mathbb{W}_{4}$ и эта точка аналитически изоморфна точке

$$
\left\{x_{1} x_{2}+x_{3}^{2}+x_{4}^{n}=0\right\} / \boldsymbol{\mu}_{2}(1,1,1,0), \quad n \geqslant 1 .
$$

ДоказАТЕльство. Если многообразие $X$ особо в некоторой точке $P \in S$, то и поверхность $S$ особа в $P$ (поскольку $S$ - дивизор Картье). Предположим, что $S \simeq \mathbb{W}_{4}$. Тогда индекс (горенштейновости) вершины $O \in S$ равен индексу точки $O \in X$. Поэтому $O \in X$ - точка индекса 2 (в частности, она особа). Теперь рассмотрим $X \ni O$ как малую окрестность и рассмотрим каноническое $\boldsymbol{\mu}_{2}$-накрытие $X^{\prime} \rightarrow X$ вблизи $O$. Оно индуцирует накрытие $S^{\prime} \rightarrow S$, где $S^{\prime} \simeq \mathbb{C}^{2} / \boldsymbol{\mu}_{2}(1,1,1)$, т.е. $S^{\prime}$ - особенность типа $A_{1}$. Так как $S^{\prime} \subset X^{\prime}$ - дивизор Картье, мы можем считать, что $X^{\prime} \ni O^{\prime}$ задается многочленом $x_{1} x_{2}+x_{3}^{2}+x_{4} \phi\left(x_{1}, x_{2}, x_{3}, x_{4}\right)$ и $\boldsymbol{\mu}_{2}$ действует на $x_{1}, x_{2}, x_{3}$ по правилу $x_{i} \rightarrow-x_{i}$. Остальное теперь следует из классификации терминальных особенностей. 
Лемма 8.9. Пусть $X \ni O$ - особая точка вида (8.2), пусть $\sigma: \widetilde{X} \rightarrow X$ - взвешенное раздутие с весами $\frac{1}{2}(1,1,1,2)$, и пусть $E$ - исключительный дивизор. Тогда

(i) $E \simeq \mathbb{W}_{4}$ nрu $n \geqslant 2$ u $E \simeq \mathbb{P}^{2}$ npu $n=1$;

(ii) $a(E)=\frac{1}{2}$;

(iii) если $n=1$, то многообразие $\widetilde{X}$ неособо, если $n \geqslant 2$, то $\widetilde{X}$ имеет ровно одну особую точку в вершине $O_{4} \in \mathbb{W}_{4}$, которая (с точностью до аналитического изоморфизма) имеет вид

$$
\left\{x_{1} x_{2}+x_{3}^{2}+x_{4}^{n-1}=0\right\} / \boldsymbol{\mu}_{2}(1,1,1,0) .
$$

ДокаЗАТЕЛЬство. Дивизор $E$ определяется в $\mathbb{P}(1,1,1,2)$ уравнением $x_{1} x_{2}+$ $x_{3}^{2}=0$ при $n \geqslant 2$ и уравнением $x_{1} x_{2}+x_{3}^{2}+x_{4}^{2}=0$ при $n=1$. Отсюда следует п. (i). Вычисление дискрепантности в п. (ii) - стандартная торическая техника. Докажем п. (iii). Многообразие $\widetilde{X}$ покрывается четырьмя аффинными картами $U_{i}$. Отображение $X \rightarrow U_{1} \simeq \mathbb{C}^{4}$ задается следующими формулами:

$$
\left(x_{1}, x_{2}, x_{3}, x_{4}\right) \longrightarrow\left(x_{1}^{1 / 2}, x_{2} x_{1}^{1 / 2}, x_{3} x_{1}^{1 / 2}, x_{4} x_{1}\right) .
$$

В этой карте $\widetilde{X}=\left\{x_{2}+x_{3}^{2}+x_{4}^{n} x_{1}^{n-1}\right\}$ неособо. Вычисления для $U_{2}$ и $U_{3}$ полностью аналогичны.

Отображение $X \rightarrow U_{4} \simeq \mathbb{C}^{4} / \boldsymbol{\mu}_{2}(1,1,1,0)$ имеет вид

$$
\left(x_{1}, x_{2}, x_{3}, x_{4}\right) \longrightarrow\left(x_{1} x_{4}^{1 / 2}, x_{2} x_{4}^{1 / 2}, x_{3} x_{4}^{1 / 2}, x_{4}\right) .
$$

Таким образом, в этой карте $\widetilde{X}=\left\{x_{1} x_{2}+x_{3}^{2}+x_{4}^{n-1}\right\} / \boldsymbol{\mu}_{2}(1,1,1,0)$ имеет ровно одну особую точку в начале координат. Остальное очевидно.

Чтобы закончить доказательство предложения 8.4, рассмотрим взвешенное раздутие $\sigma: \widetilde{X} \rightarrow X$ как в лемме 8.9. Тогда

$$
K_{\widetilde{X}}+\widetilde{S}=\sigma^{*}\left(K_{X}+S\right)-\frac{1}{2} E, \quad \sigma^{*} S=\widetilde{S}+E
$$

(поскольку $S$ - дивизор Картье и пара $(X, S)$ чисто логтерминальна). Следовательно, пара $\left(\widetilde{X}, \widetilde{S}+\frac{1}{2} E\right)$ также чисто логтерминальна. Отсюда $\widetilde{S}-$ нормальная поверхность. Заметим, что дивизор

$$
K_{\widetilde{S}}=\left.\left(K_{\widetilde{X}}+\widetilde{S}\right)\right|_{\widetilde{S}}=-\left.\frac{1}{2} E\right|_{\widetilde{S}}+\left.\sigma^{*}\left(K_{X}+S\right)\right|_{\widetilde{S}}
$$

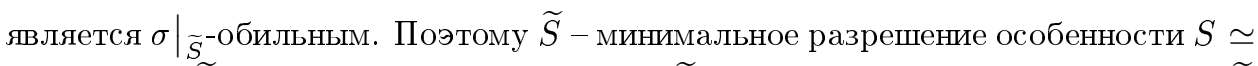
$\mathbb{W}_{4}$ и тогда $\widetilde{S} \simeq \mathbb{F}_{4}$. Так как поверхность $\widetilde{S}$ неособа, неособо и многообразие $\widetilde{X}$ вдоль $\widetilde{S}$. Ясно, что $\widetilde{S} \cap E$ является минимальным сечением $\Sigma$ на $\widetilde{S} \simeq \mathbb{F}_{4}$. Пусть 
$l$ - слой проекции $\widetilde{S} \simeq \mathbb{F}_{4} \rightarrow \mathbb{P}^{1}$. Заметим, что пересечение $\widetilde{S} \cap E$ приведено в обшей точке (поскольку пара $\left(\widetilde{X}, \widetilde{S}+\frac{1}{2} E\right)$ чисто логтерминальна). Тогда $\widetilde{S} \cdot l=\sigma^{*} S \cdot l-E \cdot l=-\Sigma \cdot l=-1$. Согласно критерию стлгиваемости существует бирациональное стягивание $\varphi: \widetilde{X} \rightarrow X_{n-1}$ поверхности $\widetilde{S}$ над $Z$ такое, что кривая $\varphi(\widetilde{S})$ содержится в множестве неособых точек многообразия $X_{n-1}$. Многообразие $X_{n-1}$ имеет ровно одну особую точку, и эта точка имеет вид (8.3). Продолжая процесс, мы получим следуюшую серию бирациональных преобразований над $Z$ :

$$
X=X_{n} \rightarrow X_{n-1} \rightarrow \cdots \rightarrow X_{1} \rightarrow X_{0} .
$$

На последнем шаге многообразие $X_{0}$ будет неособым. Таким образом, $X_{0} \rightarrow Z-$ $\mathbb{P}^{2}$-расслоение. Это доказьвает предложение 8.4.

\section{§9. Случай, когда $X_{\eta} \simeq \mathbb{P}^{1} \times \mathbb{P}^{1}$}

ПРЕДПолоЖЕНИя 9.1. В этом параграфе мы предположим, что $f: X \rightarrow Z-$ экстремальное стягивание Мори с общим слоем - $X_{\eta} \simeq \mathbb{P}^{1} \times \mathbb{P}^{1}$. Пусть $H \in \mathscr{H}_{X}-$ обший дивизор.

Лемма 9.2 (ср. [26; 3.5]). Существует вложение $X$ в $\mathbb{P}^{3}$-расслоение над $Z$ такое, что каждый слой $X_{\eta}$ является приведенной неприводимой квадрикой (в частности, $X$ горенштейново и каждый дивизор Вейля на $X$ является дивизором Картье).

НАБРОСОК ДОКАЗАТЕЛЬСТВА. Пусть $f^{*} z_{0}$ - произвольньЙ слой. Полностью аналогично доказательствам леммы 8.5 и следствия 8.6 можно показать, что $f^{*} z_{0}-$ неприводимая приведенная нормальная поверхность, изоморфная $\mathbb{P}^{1} \times \mathbb{P}^{1}$ или квадратичному конусу $\mathbb{W}_{2}$. Поэтому дивизор $H$ относительно очень обилен над $Z$ и задает требуемое вложение.

ОБознАчения 9.3. Таким образом, имеется вложение $X \hookrightarrow \mathbb{P}$ над $Z$, где

$$
\mathbb{P}=\mathbb{P}(\mathscr{E}), \quad \mathscr{E}=\bigoplus_{i=1}^{4} \mathscr{O}_{\mathbb{P}^{1}}\left(d_{i}\right)
$$

и $X_{\eta} \subset \mathbb{P}_{\eta}$ - квадрика. Положим $d=\sum d_{i}$. Мы можем выбрать $d_{i}$ так, что $d_{1} \geqslant d_{2} \geqslant d_{3} \geqslant d_{4}=0$. Пусть $M-$ тавтологический дивизор на $\mathbb{P}$ и $F-$ слой проекции $\pi: \mathbb{P} \rightarrow Z$.

Будет доказано следующее

ПРЕДЛОЖЕНИЕ 9.4. Если в обозначениях $9.3 \operatorname{dim}\left|-K_{X}\right| \geqslant 38$, то существует $\left(K_{X}+\mathscr{L}\right)$-крепантное бирациональное отображение $X$ на $\mathbb{P}^{2}$-расслоение над $Z$.

Как и в $\S 8$, этого достаточно для доказательства теоремы 1.5 в случае $X_{\eta} \simeq$ $\mathbb{P}^{1} \times \mathbb{P}^{1}$. Напомним хорошо известные факты, которые получаются из формулы Хирша и относительной точной последовательности Эйлера. 
Лемма 9.5. Имеют место следующие соотношения:

(i) $\operatorname{Pic} \mathbb{P}=\mathbb{Z} \cdot M \oplus \mathbb{Z} \cdot F$;

(ii) $M^{4}=d, \quad M^{3} \cdot F=1, F^{2} \equiv 0$;

(iii) $-K_{\mathbb{P}}=4 M+(2-d) F$.

Таким образом, $X \sim 2 M+r F$ для некоторого $r \in \mathbb{Z}$. Положим $G:=\left.M\right|_{X}$ и $Q:=\left.F\right|_{X}$. По формуле присоединения

$$
-K_{X}=2 G+(2-d-r) Q
$$

СЛЕДСТВИЕ 9.6. В обозначениях, приведенных выше, имеем $\operatorname{Pic} X \simeq \mathbb{Z} \cdot G \oplus$ $\mathbb{Z} \cdot Q$.

ЛЕмма 9.7. В доказательстве предложения 9.4 мы можем считать, ито $d+r \geqslant 3$.

ДокАЗАТЕЛЬСТво. Если $d+r<2$, то дивизор $-K_{X}$ обилен (поскольку $|G|$ и $|Q|$ - линейные системы без базисных точек и неподвижных компонент). По классификации трехмерных неособых многообразий Фано имеем

$$
\operatorname{dim}\left|-K_{V}\right| \leqslant \operatorname{dim}\left|-K_{X}\right| \leqslant 34
$$

Пусть теперь $d+r=2$. Тогда $-K_{X}=2 G$. В этой ситуации общий элемент линейной системы $|G|$ имеет лишь дювалевские особенности (см., например, [18]). По формуле присоединения антиканонический дивизор $-K_{G}$ численно эффективен и объемен. Так как $\operatorname{dim}\left|-K_{X}\right| \geqslant 38$, то, как и в $(2.1)$, имеем $-K_{X}^{3} \geqslant 72$. Отсюда $K_{G}^{2}=-\frac{1}{8} K_{X}^{3} \geqslant 9$. С другой стороны, применяя формулу Нётера к минимальному разрешению $\widetilde{G} \rightarrow G$, получим $K_{G}^{2} \leqslant 9$, причем равенство имеет место, только если $G=\widetilde{G} \simeq \mathbb{P}^{2}$ (ср. с идеей доказательства теоремы 1.5 в $\left.\S 1\right)$. Но последнее дает нам противоречие, поскольку тогда поверхность $G$ невозможно сюръективно отобразить на кривую.

Мы можем записать $-K_{X} \sim H+B$, где $B$ - эффективньй дивизор. По следствию $9.6 H \sim G+\alpha Q$ и $B \sim G-(d+r+\alpha-2) Q$ для некоторого $\alpha \in \mathbb{Z}$.

ЛЕмма 9.8. В доказательстве предложсения 9.4 мы можем считать, что $d+2 r \leqslant 6$.

ДоказАТЕЛЬСтво. Так как дивизор $H$ численно эффективен, то

$$
0 \leqslant\left(-K_{X}\right) \cdot B \cdot H=2(6-d-2 r)
$$

что и доказывает утверждение.

ЛЕмма 9.9. В доказательстве предложения 9.4 мы можем считать, что $r<0$. 
ДокаЗАТЕЛЬСТво. Пусть $r \geqslant 0$. Тогда $d \leqslant 6$. Так как $R^{i} f_{*}\left(\mathscr{O}_{X}(H)\right)=0$ при $i>0$ (см. лемму 4.4), то

$$
h^{0}\left(\mathscr{O}_{X}(H)\right)=h^{0}\left(\mathscr{O}_{\mathbb{P}^{1}}(\mathscr{E}(\alpha))\right) .
$$

Если $\alpha \leqslant 0$, то $h^{0}\left(\mathscr{O}_{X}(H)\right) \leqslant h^{0}\left(\mathscr{O}_{\mathbb{P}^{1}}(\mathscr{E})\right)=d+4 \leqslant 10$. Если же $\alpha \geqslant 0$, то из того, что $B$ эффективен, получаем $\alpha \leqslant 2+d_{1}-d-r$ и

$$
h^{0}\left(\mathscr{O}_{X}(H)\right)=d+4+4 \alpha \leqslant 12+4 d_{1}-3 d-4 r \leqslant 24 .
$$

В обоих случаях мы имеем противоречие с нашим неравенством (6.3).

Линейная система $|M|$ не имеет неподвижных компонент и базисных точек и задает бирациональное стягивание $\mathbb{P}$ на конус в $\mathbb{P}^{d+3}$. В вершину конуса стягивается подмногообразие $C:=\mathbb{P}\left(\oplus_{d_{i}=0} \mathscr{O}_{\mathbb{P}^{1}}\left(d_{i}\right)\right)$. Это подмногообразие заметается кривыми $\Gamma$ такими, что $M \cdot \Gamma=0$. Так как $r<0$, то $C$ содержится в $X$. Если $\operatorname{dim} C=2$, то мы имеем противоречие с $\rho(X / Z)=1$. Поэтому $C$-кривая.

ДоКАЗАТЕЛЬСТВо ПРЕДЛОЖЕНИЯ 9.4. Так Как $d+r>2$, то мы имеем $-K_{X}$. $C<0$. Поэтому $C \subset \mathrm{Bs}\left|-K_{X}\right|$. Пусть $\sigma:(\widetilde{\mathbb{P}} \supset \widetilde{X}) \rightarrow(\mathbb{P} \supset X)-$ раздутие $C$. Тогда $-K_{\widetilde{X}}$ - обильный над $X$ дивизор Картье и исключительный дивизор $E \subset \widetilde{X}$ стягивания $\sigma: \widetilde{X} \rightarrow X$ неприводим. Так как многообразие $\widetilde{X}$ неособо в коразмерности 1 , оно нормально. Ясно, что $a(E, \mathscr{H})=0$, поэтому стягивание $\sigma$ крепантно: $K_{\tilde{X}}+\mathscr{H}=\sigma^{*}\left(K_{X}+\mathscr{H}\right)$. Таким образом, $X^{\prime}$ имеет лишш $\mathbb{Q}$-факториальные канонические особенности и $\rho\left(X^{\prime} / Z\right)=2$. Заметим, что дивизор $\sigma^{*} G-E$ численно эффективен над $Z$ (т.е. он численно эффективен на слоях $f \circ \sigma: \widetilde{X} \rightarrow Z$ ). Общий слой $\widetilde{Q}$ морфизма $f \circ \sigma$ изоморфен раздутию точки на $Q \simeq \mathbb{P}^{1} \times \mathbb{P}^{1}$. Поэтому на $\widetilde{Q}$ имеются ровно две кривые $\widetilde{C}_{1}, \widetilde{C}_{2}$ с нулевым индексом пересечения с дивизором $\sigma^{*} G-E-$ собственные прообразы образующих $C_{1}, C_{2}$, проходящих через точку $Q \cap C$. Следовательно, дивизор $\sigma^{*} G-E$ высекает экстремальный луч на $\overline{\mathrm{NE}}(\widetilde{X} / Z)$ и линейная система $\left|n\left(\sigma^{*} G-E\right)\right|$ при $n \gg 0$ задает экстремальное бирациональное стягивание $\psi: \widetilde{X} \rightarrow X^{\prime}$ над $Z$. Морфизм $\psi$ стягивает дивизор, заметаемый $\widetilde{C}_{i}$, на кривую. Отсюда видно, что общий слой морфизма $X^{\prime} \rightarrow Z$ изоморфен $\mathbb{P}^{2}$. Далее, мы можем записать $\sigma^{*} G-E=\psi^{*} G^{\prime}$ для некоторого обильного над $Z$ дивизора Картье $G^{\prime}$ на $X^{\prime}$. Пусть $Q^{\prime}$ - произвольный слой морфизма $X^{\prime} \rightarrow Z$. Тогда $\left(G^{\prime}\right)^{2} \cdot Q^{\prime}=\left(\sigma^{*} G-E\right)^{2} \cdot \widetilde{G}=1$. Как и в $\S 8$ доказьвается, что $Q^{\prime} \simeq \mathbb{P}^{2}$. Следовательно, $X^{\prime} \rightarrow Z-\mathbb{P}^{2}$-расслоение. Так как $C \subset \mathrm{Bs}\left|-K_{X}\right|$, то отображение $X \rightarrow X^{\prime}$ крепантно.

\section{§10. Случай, когда $\operatorname{dim} Z=2$}

В этом параграфе мы предположим, что $Z$ - поверхность. Доказательство следующей леммы - частный случай доказательства теоремы 11.8 в [35]. Пусть $H-$ общий элемент линейной системы $\mathscr{H}_{X}$.

Лемма 10.1. Поверхность $Z$ неособа, и стягивание $f: X \rightarrow Z$ является $\mathbb{P}^{1}$-расслоением. 
ДокаЗАТЕЛЬСТво. Если $C_{\text {gen }}-$ общий слой, то $C_{\text {gen }} \simeq \mathbb{P}^{1},-K_{X} \cdot C_{\text {gen }}=2$ и $H \cdot C_{\text {gen }}=1$. Пусть $C=f^{-1}(P)_{\mathrm{red}}$ - произвольньй слой с приведенной структурой, и пусть $m:=H \cdot C$. Для обильного дивизора $A$ на $Z$ и $a, b \gg 0$ рассмотрим общий дивизор $S \in\left|a H+b f^{*} A\right|$. По теореме Бертини $S$ - неособая поверхность, пересекаюшая $C$ трансверсально в $m a$ точках. Следовательно, вблизи $C$ ограничение $\left.f\right|_{S}: S \rightarrow Z$ является конечным морфизмом степени $m a \geqslant a$. С другой стороны, степень $\left.f\right|_{S}$ в точности равна $a$ (поскольку $S$ пересекает общий слой в $a$ точках). Поэтому морфизм $\left.f\right|_{S}$ этален вблизи $C$ и точка $P \in Z$ неособа. Отсюда немедленно получаем, что морфизм $f$ плоский (см. [37; 23.1]), а каждьй слой $f^{-1}(P)$ приведен и неприводим и является кривой арифметического рода 0 . Отсюда $f^{-1}(P) \simeq \mathbb{P}^{1}$ (в частности, морфизм $f$ гладкий).

Поверхность $Z$ рациональна (например потому, что $H^{1}\left(Z, \mathscr{O}_{Z}\right)=H^{1}\left(X, \mathscr{O}_{X}\right)=$ 0 и она накрывается компонентой дивизора $B$ - бирационально линейчатой поверхностью, см. замечание 6.5). Положим $\mathscr{E}:=f_{*} \mathscr{O}_{X}(H)$. Тогда $\mathscr{E}-$ векторное расслоение ранга 2 и $X \simeq \mathbb{P}(\mathscr{E})$.

Мы зафиксируем следуюшие обозначения и соглашения:

ОБОЗНАЧЕНИЯ И ПРЕДПОЛОЖЕНИЯ 10.2. Пусть $Z$ - неособая рациональная поверхность и $X$ - проективизация векторного расслоения $\mathscr{E}$ ранга 2 над $Z$ такие, что пара $\left(X, \mathscr{L} \subset\left|-K_{X}\right|\right)$ канонична. Предположим, что имеет место разложение $-K_{X} \sim H+B$, где $H$ и $B$ - эффективные $f$-обильные дивизоры (сечения $f$ ), дивизор $H$ численно эффективен, а отображение $\Phi_{|H|}$, заданное линейной системой $|H|$, имеет трехмерный образ. Предположим также, что $(X, \Theta)$ является порождающей 0 -парой для некоторой границы $\Theta$.

ПРЕДЛОЖЕНИЕ 10.3. В обозначениях 10.2 имеем $\operatorname{dim}|H| \leqslant 37$. Более того, если $\operatorname{dim}|H| \geqslant 36$, то $\operatorname{dim}\left|-K_{X}\right|=38$ и антиканонический образ $X$ взвешенное проективное пространство $\mathbb{P}(3,1,1,1)$.

Предположим, что поверхность $Z$ содержит (-1)-кривую. Воспользуемся обозначениями леммы 5.4. По этой лемме мы можем построить последовательность бирациональных перестроек

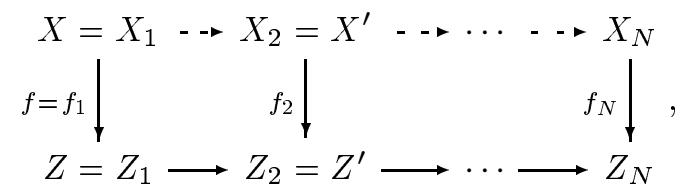

где каждый квадрат - одна из перестроек пाп. (iii)-(v) из леммы 5.4. В случае (v) мы полагаем $Z_{i}=Z_{i+1}$. Если на некотором шаге инвариант $n \geqslant 2$, то мы применяем преобразование п. (v), понижающее значение $n$. Если же $n=0$ или 1 , то мы применяем преобразования п. (iii) или п. (iv) соответственно. В этих случаях $(-1)$-кривая на базе стягивается и наша последовательность обрьвается (т.е. это возможно только на последнем шаге). 
ЛЕмма 10.4. В обозначениях, приведенных выше, для многообразия $X_{N} / Z_{N}$ выполнены все условия 10.2, причем $\operatorname{dim}\left|H_{N}\right| \geqslant \operatorname{dim}|H|$.

Однако отметим, что свойство каноничности пары $(X,|H|)$ не сохраняется при наших бирациональных преобразованиях.

ДокаЗАтЕЛЬСтво. При $n \leqslant 1$ отображение $X^{\prime} \rightarrow X$ не стягивает дивизоров. По лемме 3.1 пара $\left(X^{\prime}, \mathscr{L}^{\prime}\right)$ канонична. При $n \geqslant 2$ мы имеем $K_{X} \cdot \Sigma<0$. Поэтому $\Sigma \subset$ Bs $\mathscr{L}$ и морфизм $\sigma$ крепантен. В этом случае пара $\left(X^{\prime}, \mathscr{L}^{\prime}\right)$ канонична по лемме 3.3. Таким образом, каноничность пары $(X, \mathscr{L})$ сохраняется.

Пусть $H_{N}$ и $B_{N}-$ собственные образы $H$ и $B$ на $X_{N}$. Отметим, что отображение $\psi: X_{1} \rightarrow X_{N}$ является изоморфизмом на $X_{1} \backslash D$, а $\psi(D)$ содержится в слое $\Gamma$ проекции $f_{N}: X_{N} \rightarrow Z_{N}$. Отсюда легко видеть, что $-K_{X_{N}} \sim H_{N}+B_{N}$. Рассмотрим "домик Хиронаки"

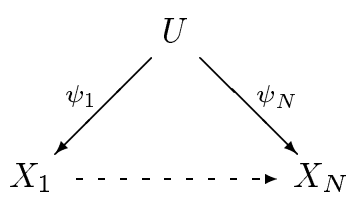

и запишем

$$
H_{U}=\psi_{1}^{*} H-\sum a_{i} E_{i}=\psi_{N}^{*} H_{N}-\sum b_{i} E_{i}-d D_{U},
$$

где $H_{U}$ и $D_{U}$ - собственные прообразы $H_{1}$ и $D$ на $U, E_{i}$ - простые дивизоры, являюшиеся исключительными как для $\psi_{1}$, так и для $\psi_{N}$, а $a_{i}, b_{i}, d \geqslant 0$. Тогда дивизор

$$
\psi_{1}^{*} H-\psi_{N}^{*} H_{N}=\sum\left(a_{i}-b_{i}\right) E_{i}-d D_{U}
$$

численно эффективен над $X_{N}$. Согласно $[22 ; 1.1]$ имеем $a_{i} \leqslant b_{i}$ и $d \geqslant 0$. Пусть $C-$ неприводимая кривая на $X_{N}$, и пусть $\widetilde{C} \subset U$ - неприводимая кривая, доминирующая $C$. Если $C=\Gamma$, то $H_{N} \cdot C>0$ (поскольку $\rho\left(X_{N} / Z_{N}\right)=1$ ). Предположим, что $C \neq \Gamma$. Так как $\psi_{N}\left(E_{i}\right), \psi_{N}\left(D_{U}\right) \subset \Gamma$, то $E_{i} \cdot \widetilde{C} \geqslant 0$ и $D_{U} \cdot \widetilde{C} \geqslant 0$. Отсюда

$$
\psi_{N}^{*} H_{N} \cdot \widetilde{C}=\psi_{1}^{*} H \cdot \widetilde{C}+\sum\left(b_{i}-a_{i}\right) E_{i} \cdot \widetilde{C}+d D_{U} \cdot \widetilde{C} \geqslant 0
$$

Это доказывает численную эффективность дивизора $H_{N}$. Лемма доказана.

СлЕДСТВИЕ 10.5. В обозначениях 10.2 мы можем считать, что $Z \simeq$ $\mathbb{P}^{2}$ uлu $\mathbb{F}_{e}, e \neq 1$.

ДоКАЗАТЕЛЬСтво. Если $Z$ содержит (-1)-кривую $C$, то по леммам 5.4 и 10.4 мы можем ее стянуть с сохранением всех условий 10.2 .

ЛЕмма 10.6. Пусть $Г \subset Z$ - неособая рациональная кривая такая, что $\operatorname{dim}|\Gamma|>0$, и пусть $\left.\mathscr{E}\right|_{\Gamma} \simeq \mathscr{O}_{\mathbb{P}^{1}}\left(d_{1}\right)+\mathscr{O}_{\mathbb{P}^{1}}\left(d_{2}\right)$. Тогда

$$
\left|d_{1}-d_{2}\right| \leqslant 2+\Gamma^{2}
$$


ДокАЗАТЕЛЬСТВо. Пусть $m=\left|d_{1}-d_{2}\right|$ и $G:=g^{-1}(\Gamma)$. Тогда $G \simeq \mathbb{F}_{m}$. Имеем

$$
-2+m=-2-\Sigma^{2}=K_{G} \cdot \Sigma=K_{X} \cdot \Sigma+G \cdot \Sigma=K_{X} \cdot \Sigma+\Gamma^{2} .
$$

Так как линейная система $\left|-K_{X}\right|$ не имеет неподвижных компонент, то $K_{X} \cdot \Sigma \leqslant 0$ и $m \leqslant 2+\Gamma^{2}$.

Далее мы предположим, что $\operatorname{dim}|H| \geqslant 36$, т.е. $h^{0}(Z, \mathscr{E})=\chi(\mathscr{E}, Z) \geqslant 37$ (см. лемму 4.4). Для краткости положим $c_{i}:=c_{i}(\mathscr{E}), i=1,2$. Тогда

$$
\begin{gathered}
-K_{X}=2 H+f^{*}\left(-K_{Z}-c_{1}\right), \\
H^{2}=H \cdot f^{*} c_{1}-f^{*} c_{2}, \quad H^{3}=c_{1}^{2}-c_{2} .
\end{gathered}
$$

Напомним теорему Римана-Роха для векторного расслоения ранга 2 над (рациональной) поверхностью $Z$ :

$$
\chi(\mathscr{E})=\frac{1}{2}\left(c_{1}^{2}-2 c_{2}-K_{Z} \cdot c_{1}\right)+2 .
$$

Так как $L \cap B$ - эффективный 1-цикл, то для любого численно эффективного дивизора $N$ на $Z$ имеем

$$
\begin{aligned}
0 & \leqslant-K_{X} \cdot B \cdot f^{*} N=\left(2 H+f^{*}\left(-K_{Z}-c_{1}\right)\right) \cdot\left(H+f^{*}\left(-K_{Z}-c_{1}\right)\right) \cdot f^{*} N \\
& =2 H^{2} \cdot f^{*} N+3\left(-K_{Z}-c_{1}\right) \cdot N=2 c_{1} \cdot N+3\left(-K_{Z}-c_{1}\right) \cdot N \\
& =-3 K_{Z} \cdot N-c_{1} \cdot N .
\end{aligned}
$$

Отсюда

$$
c_{1} \cdot N \leqslant-3 K_{Z} \cdot N
$$

Случай $Z \simeq \mathbb{P}^{2}$. Учитывая канонические изоморфизмы $H^{2 i}\left(\mathbb{P}^{2}, \mathbb{Z}\right) \simeq \mathbb{Z}$, будем считать, что $c_{1}$ и $c_{2}$ - целые числа. Неравенство (10.3) дает нам, что $0 \leqslant c_{1} \leqslant 9$, и согласно (10.1)

$$
c_{1}^{2}+3 c_{1}-2 c_{2} \geqslant 70 .
$$

СлЕДСТвИЕ 10.7. Если $\mathscr{E}$ разложимо, то $\mathscr{E} \simeq \mathscr{O}(3) \oplus \mathscr{O}(6) u \operatorname{dim}\left|-K_{X}\right|=38$.

ДоказАТельство. Пусть $\mathscr{E} \simeq \mathscr{O}(d) \oplus \mathscr{O}(d+m)$, где $m \geqslant 0$. Тогда $d \geqslant 0$ (поскольку расслоение $\mathscr{E}$ численно эффективно). Так как $c_{1}=2 d+m$ и $c_{2}=$ $d^{2}+d m \geqslant 0$, то

$$
2 d^{2}+2 d m+m^{2}+6 d+3 m \geqslant 70 .
$$

Учитывая, что $m \leqslant 3$ (лемма 10.6) и $0 \leqslant 2 d+m \leqslant 9$, получим $d=m=3$.

Таким образом, $\mathscr{L}_{X}=\left|-K_{X}\right|$ и $\Phi_{\mathscr{L}_{X}}(X)=\mathbb{P}(3,1,1,1)$, если расслоение $\mathscr{E}$ разложимо. Далее предположим, что $\mathscr{E}$ неразложимо. Мы утверждаем, что $c_{1} \leqslant 8$. Действительно, если $c_{1}=9$, то имеют место равенства в (10.3) и (10.2). Таким образом, $-K_{X} \cdot B \cdot f^{*} N=0$ для любого обильного дивизора $N$ на $\mathbb{P}^{2}$. Тогда для 
общего дивизора $L \in\left|-K_{X}\right|$ пересечение $L \cap B$ состоит из слоев $f$. Следовательно, дивизор $-K_{X}$ численно эффективен (иначе сушествует горизонтальная кривая $R$ такая, что $L \cdot R<0$ и $B \cdot R=(L-H) \cdot R<0)$. Пусть $B_{0}$ - горизонтальная компонента $B$. Легко видеть, что $-K_{X} \cdot B_{0} \cdot f^{*} N=-K_{X} \cdot\left(B-B_{0}\right) \cdot f^{*} N=0$. Поэтому $B=B_{0}$ и дивизор $B$ стягивается морфизмом $\Phi_{\left|-n K_{X}\right|}$ В точку. В этом случае расслоение $\mathscr{E}$ разложимо. Противоречие показывает, что $c_{1} \leqslant 8$.

Деление на случаи. Имеются две возможности:

а) $c_{1}$ нечетно. Положим $c_{1}=2 m-3$. Тогда $2 \leqslant m \leqslant 5$ и $2 m^{2}-3 m \geqslant 35+c_{2}$. Легко видеть, что

$$
c_{1}(\mathscr{E}(-m))=-3, \quad c_{2}(\mathscr{E}(-m))=c_{2}-m^{2}+3 m \leqslant m^{2}-35<0 .
$$

б) $c_{1}$ четно. Положим $c_{1}=2 m-2$. Тогда $1 \leqslant m \leqslant 5$ и $2 m^{2}-m \geqslant 36+c_{2}$. Здесь

$$
c_{1}(\mathscr{E}(-m))=-2, \quad c_{2}(\mathscr{E}(-m))=c_{2}-m^{2}+2 m \leqslant m^{2}+m-36<0 .
$$

В обоих случаях по теореме Римана-Роха и двойственности Серра имеем

$$
h^{0}(\mathscr{E}(-m))+h^{0}\left(\mathscr{E}(-m) \otimes \operatorname{det} \mathscr{E}(-m)^{*} \otimes \mathscr{O}(-3)\right) \geqslant \chi(\mathscr{E}(-m)) \geqslant 1 .
$$

Следовательно, $H^{0}(\mathscr{E}(-m)) \neq 0$. Пусть $s \in H^{0}(\mathscr{E}(-m))$ - ненулевое сечение. Если $s$ нигде не обрашается в нуль, то имеется вложение $\mathscr{O} \hookrightarrow \mathscr{E}(-m)$ и тогда $\mathscr{E}(-m)$ разложимо. Пусть $\varnothing \neq Y \subset \mathbb{P}^{2}$ - множество нулей $s$. Так как $c_{2}(\mathscr{E}(-m))<0$, то $\operatorname{dim} Y=1$. Выберем общую прямую $\Gamma \subset \mathbb{P}^{2}$, и пусть пересечение $\Gamma \cap Y$ состоит из $k$ точек. Положим $r:=-c_{1}(\mathscr{E}(-m))(r=2$ или 3$)$. Тогда $\left.\mathscr{E}(-m)\right|_{\Gamma}=\mathscr{O}(k) \oplus$ $\mathscr{O}(-r-k)$. По лемме 10.6 имеем $2 k+r \leqslant 3$. Отсюда $k=0$ и $Y=\varnothing$. Противоречие.

Случай $Z \simeq \mathbb{F}_{e}$.

Лемма 10.8. Если $Z \simeq \mathbb{F}_{e}$, mo $e \leqslant 4$.

ДоказАтЕльство. Действительно, пусть $\left.f\right|_{L}: L \rightarrow \bar{L} \rightarrow Z$ - факторизация Штейна. Здесь $\pi: \bar{L} \rightarrow Z$ является двойным накрытием, где $\bar{L}$ - поверхность типа К3, имеюшая лишш дювалевские особенности. По формуле Гурвица $K_{\bar{L}}=\pi^{*}\left(K_{Z}+\frac{1}{2} R\right)$, где $R \in\left|-2 K_{Z}\right|-$ дивизор ветвления. Но линейная система $\left|-2 K_{\mathbb{F}_{e}}\right|$ содержит приведенньй элемент только при $e \leqslant 4$.

ОБозНАчЕния 10.9. Учитывая канонические изоморфизмы $H^{2}\left(\mathbb{F}_{e}, \mathbb{Z}\right) \simeq$ $\mathbb{Z} \cdot \Sigma \oplus \mathbb{Z} \cdot l$ и $H^{4}\left(\mathbb{F}_{e}, \mathbb{Z}\right) \simeq \mathbb{Z}$, будем считать, что $c_{1}=a \Sigma+b l$ и $c_{2}=c$, где $a, b, c$ - целые числа. Из (10.3) и (10.4) получаем, что

$$
\begin{gathered}
a=c_{1} \cdot l \leqslant-3 K_{Z} \cdot l=6, \quad b=c_{1} \cdot(\Sigma+e l) \leqslant-3 K_{Z} \cdot(\Sigma+e l)=3(2+e) \\
\chi(\mathscr{E})=-\frac{1}{2} e a(a+1)+a b+a+b-c+2 \geqslant 37
\end{gathered}
$$

Поскольку расслоение $\mathscr{E}$ численно эффективно, то $c_{1}=a \Sigma+b l$ - численно эффективный класс, а поэтому

$$
b \geqslant e a, \quad a \geqslant 0 \text {. }
$$


Положим

$$
p:=\left\lfloor\frac{a}{2}\right\rfloor+1, \quad q:=\left\lfloor\frac{b}{2}\right\rfloor+1, \quad a^{\prime}:=a-2 p, \quad b^{\prime}:=b-2 q .
$$

Тогда

$$
-2 \leqslant a^{\prime}, b^{\prime} \leqslant-1 .
$$

Рассмотрим подкрученное расслоение $\mathscr{E}^{\prime}:=\mathscr{E} \otimes \mathscr{O}_{\mathbb{F}_{e}}(-p \Sigma-q l)$. Имеем

$$
c_{1}\left(\mathscr{E}^{\prime}\right)=a^{\prime} \Sigma+b^{\prime} l, \quad c_{2}\left(\mathscr{E}^{\prime}\right)=c+e a p-a q-b p-e p^{2}+2 p q .
$$

УТВЕРЖДЕНИЕ 10.10. Имеют место соотношения:

(i) $c_{2}\left(\mathscr{E}^{\prime}\right) \leqslant-2$;

(ii) если $c_{2}\left(\mathscr{E}^{\prime}\right)=-2$, mо $a^{\prime}=-1$;

(iii) ecли $c_{2}\left(\mathscr{E}^{\prime}\right) \geqslant-3$, mo $b^{\prime}=-2$.

ДокАЗАТЕЛЬСтво. Учитывая (10.4), получим

$$
\begin{aligned}
c_{2}\left(\mathscr{E}^{\prime}\right) & \leqslant-\frac{1}{2} e a(a+1)+a b+a+b+2-37+e a p-a q-b p-e p^{2}+2 p q \\
& =-\frac{1}{4} e a^{2}-\frac{1}{2} e a+\frac{1}{2} a b+a+b-35-\frac{1}{4} e a^{2}+\frac{1}{2} a^{\prime} b^{\prime} \\
& \leqslant-\frac{1}{4} e a^{2}+(4+e) a-29+3 e+\max \left\{-e+2,-\frac{1}{4} e+1\right\} .
\end{aligned}
$$

Если $e=0$, то

$$
c_{2}\left(\mathscr{E}^{\prime}\right) \leqslant 4 a-29+2 \leqslant-3 .
$$

Более того, равенство имеет место только при $a=6$. Тогда $b=6$ и $b^{\prime}=-2$.

Пусть $e=2$. Тогда

$$
c_{2}\left(\mathscr{E}^{\prime}\right) \leqslant-\frac{1}{2} a^{2}+6 a-23+\frac{1}{2}<-3 .
$$

Пусть $e=3$. Тогда

$$
c_{2}\left(\mathscr{E}^{\prime}\right) \leqslant-\frac{3}{4} a^{2}+7 a-20+\frac{1}{4}<-3 .
$$

Наконец, рассмотрим случай $e=4$. Тогда

$$
c_{2}\left(\mathscr{E}^{\prime}\right) \leqslant-a^{2}+8 a-17-\frac{1}{4} e a^{\prime 2}+\frac{1}{2} a^{\prime} b^{\prime} \leqslant-a^{2}+8 a-17 .
$$

Предположим, что $c_{2}\left(\mathscr{E}^{\prime}\right) \geqslant-3$. Тогда $3 \leqslant a \leqslant 4$ (учитываем, что $е a \leqslant b \leqslant$ $3(2+e))$. Если $a=4$, то $a^{\prime}=-2$ и $c_{2}\left(\mathscr{E}^{\prime}\right) \leqslant-5-b^{\prime}$. Отсюда $c_{2}\left(\mathscr{E}^{\prime}\right)=-3$ и $b^{\prime}=-2$. Пусть $a=3$. Тогда $a^{\prime}=-1$ и $c_{2}\left(\mathscr{E}^{\prime}\right) \leqslant-3-b^{\prime} / 2$. Отсюда

$$
-3 \leqslant c_{2}\left(\mathscr{E}^{\prime}\right) \leqslant-48+\frac{5}{2} b-\frac{1}{2} b^{\prime} .
$$

Это возможно только при $b=18, b^{\prime}=-2$. 
УТВЕРЖДЕНИЕ 10.11. Имеем неравенство $\chi\left(\mathscr{E}^{\prime}\right)>0$.

ДоказАтельство. Положим $c^{\prime}:=c\left(\mathscr{E}^{\prime}\right)$. По теореме Римана-Роха

$$
\chi\left(\mathscr{E}^{\prime}\right)=\left(b^{\prime}-\frac{1}{2} e a^{\prime}\right)\left(a^{\prime}+1\right)+a^{\prime}-c^{\prime}+2 .
$$

Предположим, что $\chi\left(\mathscr{E}^{\prime}\right) \leqslant 0$. Учитьвая утверждение 10.10, получим

$$
\left(b^{\prime}-\frac{1}{2} e a^{\prime}\right)\left(a^{\prime}+1\right)+a^{\prime} \leqslant c^{\prime}-2 \leqslant-4 .
$$

Отсюда $a^{\prime} \neq-1$. Следовательно, $a^{\prime}=-2$ и снова по утверждению 10.10 имеем

$$
1-e \leqslant-b^{\prime}-e \leqslant c^{\prime} \leqslant-3
$$

Таким образом, $e=4, b^{\prime}=-1$ и $c^{\prime}=-3$. Это противоречит утверждению 10.10.

УТВЕРЖДЕНИЕ 10.12. Имеем неравенство $H^{0}\left(\mathscr{E}^{\prime}\right) \neq 0$.

ДокАЗАтЕльство. Предположим, что $H^{0}\left(\mathscr{E}^{\prime}\right)=0$. Согласно утверждению 10.11 имеем $H^{2}\left(\mathscr{E}^{\prime}\right) \neq 0$. По двойственности Серра

$$
H^{2}\left(\mathscr{E}^{\prime}\right)^{*} \simeq H^{0}\left(\mathscr{E}^{\prime *} \otimes \omega_{Z}\right) \simeq H^{0}\left(\mathscr{E}^{\prime} \otimes \operatorname{det} \mathscr{E}^{\prime *} \otimes \omega_{Z}\right)
$$

С другой стороны,

$$
\left(\operatorname{det} \mathscr{E}^{\prime *} \otimes \omega_{Z}\right)^{*}=\mathscr{O}_{Z}\left(\left(a^{\prime}+2\right) \Sigma+\left(b^{\prime}+(e+2)\right) l\right)
$$

и

$$
H^{0}\left(\left(\operatorname{det} \mathscr{E}^{\prime *} \otimes \omega_{Z}\right)^{*}\right) \neq 0
$$

Противоречие.

ОКончание ДокаЗАтельства теоремы 1.5. Рассмотрим ненулевое сечение $s \in H^{0}\left(\mathscr{E}^{\prime}\right)$. Если $s$ нигде не обрашается в нуль, то $\mathscr{E} \prime$ является расширением некоторого линейного расслоения $\mathscr{E} 1$ при помощи $\mathscr{O}$. Но тогда $c_{2}\left(\mathscr{E}^{\prime}\right)=c_{2}\left(\mathscr{E}_{1}\right)=0$, что противоречит утверждению 10.10 . Поэтому, как и в случае $Z=\mathbb{P}^{2}$, схема нулей сечения $s$ содержит кривую $Y$. Пусть $Y \sim q_{1} \Sigma+q_{2} l$. Тогда ограничения $\mathscr{E}^{\prime}$ на общие кривые $l \in|l|$ и $l^{\prime} \in|\Sigma+e l|$ имеют вид:

$$
\begin{aligned}
& \left.\mathscr{E}^{\prime}\right|_{l}=\mathscr{O}\left(q_{1}\right) \oplus \mathscr{O}\left(a^{\prime}-q_{1}\right), \\
& \left.\mathscr{E}^{\prime}\right|_{l^{\prime}}=\mathscr{O}\left(q_{2}\right) \oplus \mathscr{O}\left(b^{\prime}-q_{2}\right) .
\end{aligned}
$$

По лемме 10.6 имеем

$$
\begin{aligned}
& 2 q_{1}-b^{\prime} \leqslant 2 \\
& 2 q_{2}-a^{\prime} \leqslant 2 .
\end{aligned}
$$

Так как $-a^{\prime},-b^{\prime} \geqslant 1$, это дает нам противоречие. Предложение 10.3 и первая часть теоремы 1.5 доказаны. 
Теперь предположим, что $-K_{V}^{3}=72$. Тогда согласно предложению 10.3 мы можем считать, что $\operatorname{dim}|H|=35$. Это возможно только в случае (В) из $\S 6$. Как и вьше, применяя леммы 5.4 и 10.4, мы добьемся того, что поверхность $Z$ не содержит (-1)-кривых. Следовательно, $Z \simeq \mathbb{P}^{2}$ или $Z \simeq \mathbb{F}_{e}, e=0,2,3,4$.

Пусть сначала $Z \simeq \mathbb{P}^{2}$. Согласно предложению 5.2 дивизор $-K_{X}$ не может быть численно эффективным. Следовательно, существует кривая $R$ такая, что $-K_{X} \cdot R<0$ и $B \cdot R=-K_{X} \cdot R-H \cdot R<0$. Отсюда $-K_{X} \cdot B \cdot f^{*} N>0$ для любого обильного дивизора $N$ на $Z$. Из (10.2) получаем, что $c_{1} \leqslant 8$. Далее доказательство проводится полностью аналогично доказательству случаев а), б) вьшше.

Пусть теперь $Z \simeq \mathbb{F}_{e}, e=0,2,3,4$. Воспользуемся обозначениями 10.9. Если $\chi\left(\mathscr{E}^{\prime}\right)>0$, мы получаем противоречие, как и выше. Предположим, что $\chi\left(\mathscr{E}^{\prime}\right) \leqslant 0$. Как и вьше, $-K_{X} \cdot B \cdot f^{*} N>0$ для любого обильного дивизора $N$ на $Z$. Поэтому $a<6$ или $b<3(2+e)$. Используя эти неравенства, аналогично утверждению 10.10 получаем следуюшее

УТВЕРЖДЕНИЕ 10.13. Пусть $\chi(\mathscr{E}) \geqslant 36$. Тогда $c_{2}\left(\mathscr{E}^{\prime}\right) \leqslant-4$, за исключением следующих случаев:

(i) $c_{2}\left(\mathscr{E}^{\prime}\right) \leqslant-3, e=3, a=5, b=15, a^{\prime}=-1, b^{\prime}=-1$;

(ii) $c_{2}\left(\mathscr{E}^{\prime}\right) \leqslant-1, e=4, a=5, b=18, a^{\prime}=-1, b^{\prime}=-2$;

(iii) $c_{2}\left(\mathscr{E}^{\prime}\right) \leqslant-1, e=4, a=3, b=18, a^{\prime}=-1, b^{\prime}=-2$;

(iv) $c_{2}\left(\mathscr{E}^{\prime}\right) \leqslant-2, e=4, a=4, b=18, a^{\prime}=-2, b^{\prime}=-2$.

По теореме Римана-Роха

$$
0 \geqslant \chi\left(\mathscr{E}^{\prime}\right)=\left(b^{\prime}-\frac{1}{2} e a^{\prime}\right)\left(a^{\prime}+1\right)+a^{\prime}-c^{\prime}+2
$$

Отсюда нетрудно видеть, что $a^{\prime}=-2$ и $0 \geqslant \chi\left(\mathscr{E}^{\prime}\right)=-b^{\prime}-e-c^{\prime}$. Это возможно только в случае (iv) и тогда $c_{2}\left(\mathscr{E}^{\prime}\right)=-2, c_{2}(\mathscr{E})=20, \chi\left(\mathscr{E}^{\prime}\right)=0$. Таким образом, $c_{1}=4 \Sigma+18 l$. Но тогда $H^{3}=c_{1}^{2}-c_{2}=0$. Это противоречит тому, что дивизор $H$ численно эффективен и объемен. Это заканчивает доказательство теоремы 1.5 .

\section{Список литературы}

1. Alexeev V. General elephants of $\mathbb{Q}$-Fano 3-folds // Compositio Math. 1994. V. 91. P. 91-116.

2. Fano G. Sulle varieta a tre dimensioni a curve-sezioni canoniche // Acad. Ital. Mem. Cl. Sci. Fis. Mat. Nat. 1937. V. 8. P. 23-64.

3. Fano G. Su alcune varieta algebriche a tre demensioni aventi curve-sezioni canoniche // Scritti Mat. offerti a L. Berzolari., Pavia. Tip. Rossetti., 1936. P. 329-349.

4. Conte A., Murre J.P. On the definition and on the nature of the singularities of Fano threefolds // Rend. Sem. Mat. Univ. Politec. Torino. 1986. V. 44. P. 51-67.

5. Borisov A. Boundedness of Fano threefolds with log-terminal singularities of given index // J. Math. Sci. Univ. Tokyo. 2001. V. 8. № 2. P. 329-342.

6. Чельцов И. А. Ограниченность трехмерных многообразий Фано целого индекса // Матем. заметки. 1999. Т. 66. № 3. С. 445-451.

7. Namikawa Y. Smoothing Fano 3-folds // J. Algebraic Geom. 1997. V. 6. P. 307-324. 
8. Mukai S. New developments in the theory of Fano threefolds: Vector bundle method and moduli problems // Sugaku Expositions. 2002. V. 15. № 2. P. 125-150.

9. Исковских В. А. Антиканонические модели трехмерных алгебраических многообразий // Итоги науки и техники. Соврем. проблемы матем. Т. 12. М.: ВИНИТИ, 1979. С. $59-157$.

10. Чельцов И.А. Трехмерные алгебраические многообразия, обладающие дивизором с численно тривиальным каноническим классом // УМН. 1996. Т. 51. №1(307). C. $177-178$.

11. Ishii S. Quasi-Gorenstein Fano 3-folds with isolated nonrational loci // Compositio Math. 1991. V. 77. № 3. P. 335-341.

12. Conte A., Murre J.P. Algebraic varieties of dimension three whose hyperplane sections are Enriques surfaces // Ann. Scuola Norm. Sup. Pisa Cl. Sci. (4). 1985. V. 12. P. 43-80.

13. Prokhorov Yu. G. A remark on Fano threefolds with canonical Gorenstein singularities // The Fano conference. Proceedings. Torino (Italy), 29 September-5 October 2002, Univ. degli Studi di Torino / ed. A. Collino et al. Torino, 2004.

14. Kawamata $Y$. The crepant blowing-up of 3-dimensional canonical singularities and its application to the degeneration of surfaces // Ann. of Math. (2). 1988. V. 127. P. 93-163.

15. Cutkosky S. Elementary contractions of Gorenstein threefolds // Math. Ann. 1988. V. 280. P. 521-525.

16. Reid $M$. Projective morphisms according to Kawamata // Preprint, Univ. of Warwick, 1983.

17. Saint-Donat B. Projective models of K3 surfaces // Amer. J. Math. 1974. V. 96. P. 602-639.

18. Shin K.-H. 3-dimensional Fano varieties with canonical singularities // Tokyo J. Math. 1989. V. 12. P. $375-385$.

19. Jahnke P., Radloff I. Generatedness for Gorenstein Fano threefolds with canonical singularities // Preprint, 2004; math.AG/0404155.

20. Kollár J., Mori S. Birational geometry of algebraic varieties. Cambridge: Cambridge Univ. Press, 1998. (Cambridge Tracts in Mathematics. V. 134.)

21. Kollár J. Singularities of pairs // Proc. Sympos. Pure Math. 1995. V. 62. P. 221-287.

22. Шокуров В. В. Трехмерные лог-перестройки // Изв. РАН. Сер. матем. 1992. Т. 56. № 1. C. 105-201.

23. Mori S. Flip theorem and the existence of minimal models for 3-folds // J. Amer. Math. Soc. 1988. V. 1. P. 117-253.

24. Reid M. Canonical 3-folds // Journées de Géometrie Algébrique d'Angers, Juillet 1979 / Algebraic Geometry, Angers. Sijthoff \& Noordhof, 1979. P. 273-310.

25. Prokhorov Yu. G., Shokurov V. V. Toward the second main theorem on complements: from local to global // Preprint, 2000.

26. Mori S. Threefolds whose canonical bundles are not numerically effective // Ann. of Math. (2). 1982. V. 115. P. 133-176.

27. Mori S., Mukai S. Classifications of Fano 3-folds with $B_{2} \geqslant 2$. I // Algebraic and topological theories. Papers from the symposium dedicated to the memory of Dr. Takehiko Miyata. Kinosaki, October 30-November 9, 1984. Tokyo: Kinokuniya Company Ltd., 1986. P. 496-545.

28. Prokhorov Yu. G. On $\mathbb{Q}-$ Fano fiber spaces with two-dimensional base // Preprint № 95-33. Max-Planck-Institut, 1995.

29. Демин И. В. Ограниченность степени трехмерных многообразий Фано, представимых в виде расслоений на коники // УМН. 1981. Т. 36. №3. С. 203-204.

30. Саржисов В.Г. О структурах расслоений на коники // Изв. АН СССР. Сер. матем. 1982. T. 46. № 2. C. 371-408.

31. Саржисов В.Г. Бирациональные автоморфизмы расслоений на коники // Изв. АН СССР. Сер. матем. 1980. Т. 44. № 4. С. 918-945. 
32. Исковских В. А. К проблеме рациональности расслоений на коники // Матем. сб. 1991. T. 182. № 1. C. 114-121.

33. Iskovskikh V.A., Prokhorov Yu.G. Fano varieties // Encyclopaedia of mathematical sciences. Algebraic geometry. V / ed. A.N. Parshin, I. R. Shafarevich. V. 47. Berlin: Springer-Verlag, 1999.

34. Sano T. Classification of non-Gorenstein $\mathbb{Q}$-Fano $d$-folds of Fano index greater than $d-2$ // Nagoya Math. J. 1996. V. 142. P. 133-143.

35. Fujita T. Classification theories of polarized varieties. Cambridge: Cambridge Univ. Press, 1990. (London Math. Soc. Lecture Note Ser. V. 155.)

36. Fujita $T$. On the structure of polarized varieties with $\Delta$-genera zero // J. Fac. Sci. Univ Tokyo. Sec. IA. 1975. V. 22. P. 103-115.

37. Matsumura H. Commutative ring theory. Cambridge: Cambridge Univ. Press., 1986.

Московский государственный университет

им. М.В. Ломоносова

E-mail: prokhoro@mech.math.msu.su
Поступила в редакцию 02.12.2003 и 01.06.2004 